\title{
Ferroptosis-related long non-coding RNAs and the roles of LASTR in stomach adenocarcinoma
}

\author{
GONGJUN WANG ${ }^{1,2 *}$, LIBIN SUN ${ }^{2 *}$, SHASHA WANG ${ }^{2}$, JING GUO $^{2}$, \\ RUOXI XIAO $^{1}$, WENQIAN LI ${ }^{1}$, WEIWEI QI ${ }^{2}$ and WENSHENG QIU ${ }^{2}$ \\ ${ }^{1}$ Department of Medicine, Qingdao University, Qingdao, Shandong 266071; ${ }^{2}$ Department of Oncology, \\ Affiliated Hospital of Qingdao University, Qingdao, Shandong 266000, P.R. China
}

Received November 14, 2021; Accepted January 11, 2022

DOI: $10.3892 / \mathrm{mmr} .2022 .12634$

\begin{abstract}
Ferroptosis is a form of programmed cell death that participates in diverse physiological processes. Increasing evidence suggests that long noncoding RNAs (lncRNAs) regulate ferroptosis in tumors, including stomach adenocarcinoma (STAD). In the present study, RNA-sequencing data from The Cancer Genome Atlas database and ferroptosis-related markers from the FerrDb data resource were analyzed to select differentially expressed lncRNAs. Univariate and multivariate Cox regression analyses were performed on these differentially expressed lncRNAs to screen 12 lncRNAs linked with overall survival (OS) and 13 associated with progression-free survival (PFS). Subsequently, two signatures for predicting OS and PFS were established based on these lncRNAs. Kaplan-Meier analyses indicated that the high-risk group of patients with STAD had relatively poor prognosis. The areas under the receiver operating characteristic curves of the two signatures indicated their excellent efficacy in predicting STAD prognosis. In addition, the effect of the IncRNA LASTR on proliferation and migration in gastric cancer was confirmed and the relationship between LASTR and ferroptosis was initially explored through experiments. These results provide potential novel targets for tumor treatment and promote personalized medicine.
\end{abstract}

Correspondence to: Professor Wensheng Qiu or Professor Weiwei Qi, Department of Oncology, Affiliated Hospital of Qingdao University, 7 Jiaxing Road, Qingdao, Shandong 266000, P.R. China E-mail: wsqiuqd@163.com

E-mail: qwwdz@126.com

${ }^{*}$ Contributed equally

Key words: stomach adenocarcinoma, TCGA, ferroptosis, lncRNA, prognosis signature, LASTR

\section{Introduction}

Gastric cancer (GC) has the fifth-highest incidence among all cancer types and the third-highest cancer-associated mortality rate globally (1). The incidence of GC accounts for the second-highest cancer incidence in China. In 2015, $\sim 679,100$ new cases and 498,000 deaths were recorded, causing a considerable burden to society (2). As most individuals are already at the advanced stage when they are diagnosed and based on the incidences of chemotherapy resistance and recurrence, the overall five-year OS of patients is $<25 \%$ (3). Therefore, identifying its underlying pathogenic mechanism and detecting novel and reliable potential therapeutic targets is essential to enhance the prognosis of individuals with GC.

The roles of ferroptosis in tumors have attracted increasing attention recently. Ferroptosis is an iron-dependent form of programmed cell death that is different from apoptosis, cell necrosis and autophagy (4). The primary mechanism of ferroptosis depends on the action of ester oxygenase or divalent iron, which catalyzes lipid peroxidation of unsaturated fatty acids, hence triggering cell death. Besides, it also functions in the antioxidant system (the glutathione system) to regulate the reduction of the core enzyme, phospholipid hydroperoxide glutathione peroxidase 4 (GPX4) $(5,6)$. An increasing number of studies have indicated that long noncoding RNAs (lncRNAs) are able to regulate ferroptosis and mediate biological behavior in various tumors. Zhang et al (7) documented that lncRNA OIP5-antisense 1 (AS1) induced ferroptosis resistance and promoted prostate cancer progression. Ma et al (8) proved that the lncRNA MEG8 repressed proliferation and induced ferroptosis in hemangioma endothelial cells. The lncRNA long intergenic RNA (LINC)00618 was reported to accelerate ferroptosis via increasing the contents of lipid reactive oxygen species and iron in human leukemia (9). However, there is still a lack of research that systematically assesses ferroptosis-related lncRNA signatures and explains their relationship with overall survival (OS) and progression-free survival (PFS) in patients with stomach adenocarcinoma (STAD).

In the present study, two signatures of differentially expressed ferroptosis-related IncRNAs were established to evaluate OS and PFS prognosis based on The Cancer Genome Atlas (TCGA) data. Furthermore, experiments were conducted to validate the influence of a unique overlapping 
lncRNA, namely lncRNA associated with spliceosome associated factor 3, U4/U6 recycling protein (SART3) regulation of splicing (LASTR) of the signatures for PFS and OS in GC.

\section{Materials and methods}

Data collection. RNA-sequencing data of 407 patients (32 non-malignant and 375 tumors) were downloaded from the TCGA-STAD data resource (https://portal.gdc.cancer.gov/). TCGA constitutes a publicly funded project whose purpose includes cataloging and discovering significant cancer-pathogenesis genome changes in large datasets of $>30$ human cancer types via large-scale genome sequencing along with integrated multidimensional analyses. The matching TCGA clinical data were obtained from cBioPortal (http://www.cbioportal.org/) (10). The matching ferroptosis-related genes were abstracted from FerrDb (http://www.zhounan.org/ferrdb/) (11), an online consortium providing comprehensive and up-to-date data resources for ferroptosis-related biomarkers, their modulatory molecules and diseases.

Profiling differentially expressedferroptosis-related IncRNAs (DEFRLs). To determine ferroptosis-related lncRNAs, to limma R tool was employed to perform differential analyses for the STAD samples from TCGA. Significant differences in expression were determined using a false discovery rate $<0.05$ and $\mid \log 2$ (fold change) $\mid \geq 1$ as the threshold. Pearson correlation analysis was adopted to assess the relationship of the lncRNAs with ferroptosis markers. A correlation coefficient of $\left|\mathrm{R}^{2}\right|>0.45$ at $\mathrm{P}<0.05$ was considered to indicate a significant correlation.

Functional enrichment analysis of DEFRLs. The clusterprofiler R tool was employed to perform Gene Ontology (GO) coupled with Kyoto Encyclopedia of Genes and Genomes (KEGG) functional analysis to elucidate the role of unrecovered DEFRLs (12). An adjusted $\mathrm{P}<0.05$ denoted statistical significance.

Development of the ferroptosis-related IncRNA prognostic signatures. OS and PFS were analyzed to gain insight into the prognostic significance of DEFRLs in individuals with STAD. OS was defined as the time from the first day of diagnosis until death from any cause, while PFS included the time from the day of diagnosis to cancer progression or death. First, a univariate Cox analysis was adopted to explore OS- and PFS-related DEFRLs. Furthermore, multivariate Cox regression was employed to determine the potential OS- and PFS-related DEFRLs to create two predictive signatures, the OS and PFS signatures, respectively. The DEFRLs' coefficients in the final signatures were validated simultaneously and utilized to compute the risk scores for each STAD patient. And all subjects were stratified into either low-risk or high-risk groups, as per the median score. The risk score was calculated as follows:

$$
\text { Risk score }=\sum_{i=0}^{n} \beta i * G i \text {, }
$$

where $\beta i$ is the coefficient of IncRNA $i$ in the multivariate Cox analysis; $G i$ is the expression value of $\operatorname{lncRNA} i$; and $n$ is the number of lncRNAs in the signature.
To explore the efficiency of the signatures, receiver operating characteristic (ROC) analysis was performed. The 'survival ROC' tool was employed to create ROC curves at 1,3 and 5 years and the matching time-based areas under the curves (AUCs) were computed. Furthermore, the Kaplan-Meier (K-M) survival plots were generated and the log-rank test was used to assess the differences in OS and PFS between the high- and low-risk groups.

Predictive nomogram integrating DEFRL signatures and clinical variables. Clinical characteristics, including sex, age and grade, were abstracted from the cBioPortal data resource. Univariate Cox regression integrating the signature with the clinical information was performed for individuals with STAD. Factors harboring $\mathrm{P}<0.05$ were subjected to multivariate regression to determine the independent predictive factors. Subsequently, two predictive nomograms were created using the R 'rms' package on the basis of the independent predictive factors for estimating OS and PFS of individuals with STAD. The concordance index (C-index) was employed to explore the discrimination efficiency of these two nomograms.

Cells and culture conditions. The AGS and MKN7 cell lines were acquired from the cell bank of the Chinese Academy of Sciences and cultured in RPMI-1640 medium (PM 150110; Procell Life Science \& Technology Co., Ltd) enriched with $10 \%$ FBS (Gibco; Thermo Fisher Scientific, Inc.) at $37^{\circ} \mathrm{C}$ in a humidified atmosphere containing $5 \% \mathrm{CO}_{2}$.

Cell line authentication. The appropriate amount of MKN-7 cells (cat. no. PC244; $1 \times 10^{6}$ ) was processed with Chelex100 (Bio-Rad Laboratories, Inc.) to extract DNA and then the 21CELLID System (Promega Corporation) was used to amplify 20 short tandem repeat sites and sex identification sites. The AB13130x1 genetic analyzer (Applied Biosystems; Thermo Fisher Scientific, Inc.) was used for PCR product detection. GeneMapper IDX v4 software (Applied Biosystems; Thermo Fisher Scientific, Inc.) was employed to analyze the test results and compare them with entries in databases such as ATCC (American Type Culture Collection; https://www. atcc.org/), DSMZ (German Collection of Microorganisms and Cell Cultures; https://www.dsmz.de/), JCRB (Japanese Cancer Research Resources Bank; https://cellbank.nibiohn. go.jp/english/cellsearch_e/) and Cellosaurus (https://web. expasy.org/cellosaurus/).

Transfection. The cells were transfected with small interfering RNAs (siRNAs) (Table SI) targeting LASTR (siLASTR; LncRNA-Pharma) and negative control (siNC) with the Lipofectamine 2000 system (Invitrogen; Thermo Fisher Scientific, Inc.) as described by the manufacturer. Cells were incubated with LASTR siRNAs for $48 \mathrm{~h}$ and harvested for subsequent experiments.

$R N A$ isolation and reverse transcription-quantitative ( $R T-q)$ $P C R$. The TRIzol reagent was used to extract and purify total RNA from cells (Takara Bio, Inc.). cDNA was generated from the RNA via RT with Prime Script RT Master Mix (Takara Bio, Inc.), as per the manufacturer's instructions. Subsequently, qPCR was run on the ABI 7500HT Fast Real-Time PCR 
Platform (Applied Biosystems; Thermo Fisher Scientific, Inc.). Thermocycling conditions were: $95^{\circ} \mathrm{C}$ for $15 \mathrm{~min}$; 40 cycles of $95^{\circ} \mathrm{C}$ for $1 \mathrm{~min}$ and $60^{\circ} \mathrm{C}$ for $1 \mathrm{~min}$. The $2^{-\Delta \Delta \mathrm{Cq}}$ method (13) was adopted to determine relative lncRNA expression with GAPDH as the normalization control. The oligonucleotide primers for RT-qPCR included were as follows: LASTR forward, 5'-GAGAAGACAGTGGGTGAAGTCC-3' and reverse, 5'-GACTCTAGGCACCAGCTGAC-3'; and GAPDH forward, 5'-GGAAGCTTGTCATCAATGGAAATC-3' and reverse, 5'-TGATGACCCTTTTGGCTCCC-3'.

Western blot analysis. The GC cells were inoculated onto 6-cm plates for $48 \mathrm{~h}$ and harvested via scraping. Lysis was performed by applying RIPA lysis buffer enriched with protease and phosphatase inhibitors (Beijing Solarbio) for $30 \mathrm{~min}$. Subsequently, the cells were centrifuged at $12,000 \mathrm{x} \mathrm{g}$ for $20 \mathrm{~min}$ at $4^{\circ} \mathrm{C}$, and the protein was quantitated with a BCA protein assay kit (Beyotime Institute of Biotechnology, Inc.). Subsequently, $20 \mu \mathrm{g}$ of the proteins were fractionated by $12.5 \%$ SDS-PAGE and transfer-embedded onto PVDF membranes (MilliporeSigma). Blocking of the membranes was performed for $2 \mathrm{~h}$ using $5 \%$ skimmed milk (Nestlé S.A.) dispersed in Tris-buffered saline containing Tween-20 (TBST). The membranes were then inoculated overnight $\left(4^{\circ} \mathrm{C}\right)$ with the indicated primary antibody $(1: 1,000)$. Next, the membranes were rinsed in TBST for $10 \mathrm{~min}$ and then inoculated at room temperature with the secondary HRP-conjugated antibodies (1:8,000 dilution; abs20002; Absin Bioscience Inc.) for $2 \mathrm{~h}$ at room temperature. Subsequently, the membranes were rinsed with TBST and the bound antibodies were visualized with a chemiluminescence (ECL) kit (cat. no. 34095, Thermo Fisher Scientific, Inc.) on a ChemiDoc XRS+ gel imager infrared imaging Platform (Bio-Rad Laboratories, Inc.). Antibodies against $\beta$-actin (cat. no. 4970; Cell Signaling Technology, Inc.) and anti-GPX4 (cat. no. ab18196) purchased from Abcam and secondary antibodies (cat. no. abs20002) acquired from Absin Bioscience Inc. were used for the western blotting.

5-Ethynyl-2'-deoxyuridine (EdU) incorporation assay. GC cells $\left(1 \times 10^{5} /\right.$ well) were plated onto 24 -well plates, allowed to grow for $48 \mathrm{~h}$ and incubated with medium enriched with $50 \mu \mathrm{M}$ EdU (Beyotime Institute of Biotechnology) for $2 \mathrm{~h}$. The cells were fixed in 4\% paraformaldehyde (PFA; Beyotime Institute of Biotechnology) followed by permeabilization and then a click reaction mixture (cat. no. C0078S; Beyotime Institute of Biotechnology) (200 $\mu \mathrm{l} /$ well) was added with subsequent incubation for $30 \mathrm{~min}$. Nuclear staining was performed with Hoechst 33342 (200 $\mu \mathrm{l} /$ well) for $30 \mathrm{~min}$ and a fluorescence microscope (Olympus IX 51; Olympus Corporation) was employed to visualize the cells.

Colony-formation assay. Cells were transfected with siRNA for two days and then inoculated into 6-well dishes at 300 cells/well and allowed to grow for 10 days. Next, the cells were fixed in 4\% PFA for 30-60 min, followed by staining with $0.5 \%$ crystal violet for $20 \mathrm{~min}$ at room temperature. After numerous washes in $\mathrm{ddH}_{2} \mathrm{O}$, images of the colonies were acquired and their numbers determined. A colony was considered to be $>50$ cells.
Table I. Clinical characteristics of patients in The Cancer Genome Atlas-stomach adenocarcinoma dataset.

\begin{tabular}{lr}
\hline Variable & Number of samples \\
\hline Sex (male/female) & $241 / 134$ \\
Age at diagnosis ( $\leq 65 />65$ years/NA) & $164 / 207 / 4$ \\
Grade (G1/G2/G3/NA) & $10 / 137 / 219 / 9$ \\
TNM stage (I/II/III/IV/NA) & $53 / 111 / 150 / 38 / 23$ \\
TNM stage (T0/T1/T2/T3/T4/NA) & $19 / 80 / 168 / 100 / 8$ \\
N (N0/N1/N2/N3/NA) & $111 / 97 / 75 / 74 / 18$ \\
M (M0/M1/NA) & $330 / 25 / 20$
\end{tabular}

NA, information not available.

Transwell migration assay. Cell migration was evaluated using a Transwell insert (cat. no. 354480; $8.0 \mu \mathrm{m}$ pore; BD Biosciences) without Matrigel. Following transfection, $2 \times 10^{5}$ cells in serum-free medium were seeded into each upper compartment of the Transwell insert. Medium enriched with $20 \%$ FBS was added to the lower compartment and allowed to grow for one day. Subsequently, the cells were fixed with $4 \%$ PFA for $30 \mathrm{~min}$, followed by staining with $0.5 \%$ crystal violet for $20 \mathrm{~min}$ all at room temperature) and then rinsed with PBS. The cells were counted in five fields (top, bottom, center, left and right) under a microscope (Olympus IX 51; Olympus Corporation).

Wound-healing assay. The GC cells were seeded into 6-well plates to form a confluent layer and a sterile pipette tip was employed to make a linear scratch. Next, the cells were rinsed with PBS and then further cultured in a medium enriched without FBS. Images were acquired at 0 and $24 \mathrm{~h}$ with a phase-contrast microscope. The fraction of wound healing was determined as follows: [1-(empty area $24 \mathrm{~h} / \mathrm{empty}$ area 0 h)] $\times 100 \%$.

Statistical analysis. All data analyses were implemented in Bioconductor packages in $\mathrm{R}$ software, version 4.1.0, and GraphPad Prism 8.0 Software (GraphPad Software, Inc.). The unpaired Student's t-test, the Wilcoxon rank-sum test, ANOVA and the Kruskal-Wallis test were adopted to compare continuous variables. Pearson analysis was implemented for the correlation analyses. $\mathrm{P}<0.05$ was considered to indicate statistical significance.

\section{Results}

Patient characteristics. Overall, 375 STAD tumor samples along with 32 non-malignant adjacent tissues were included and their expression profiles were identified. The clinical features of the patients are provided in Table I (male: female ratio, $1.80: 1 ; \leq 65:>65$ ratio, 0.79:1). Next, 382 ferroptosis-related markers were obtained from FerrDb (Fig. 1A). Using Pearson's correlation test, 503 differentially expressed ferroptosis-related lncRNAs were obtained (Fig. 1B). To gain a profound understanding of how these ferroptosis-related lncRNAs may drive STAD development, GO along with KEGG enrichment 
A

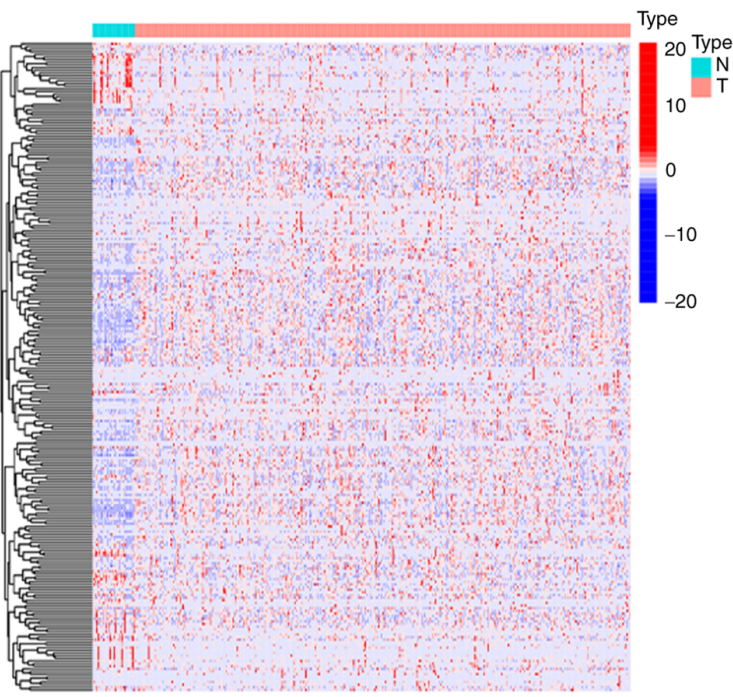

B

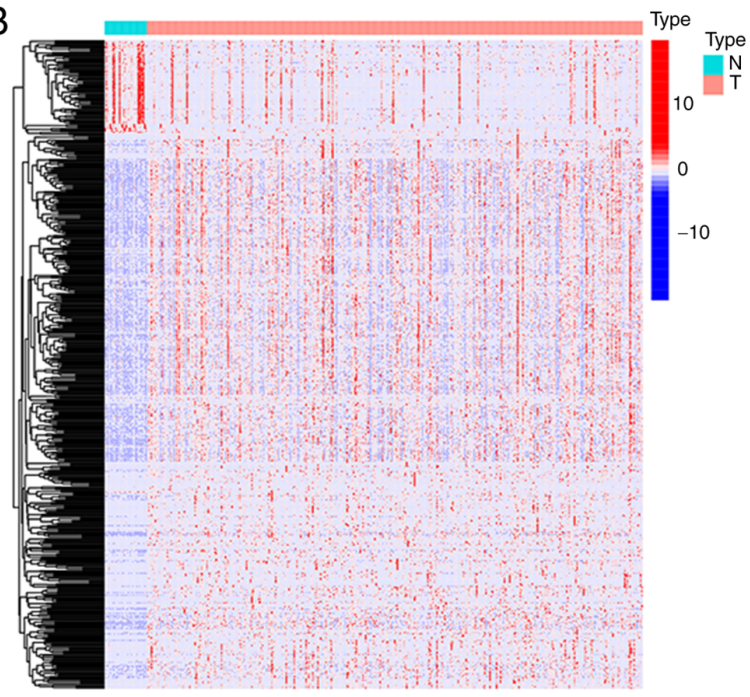

C

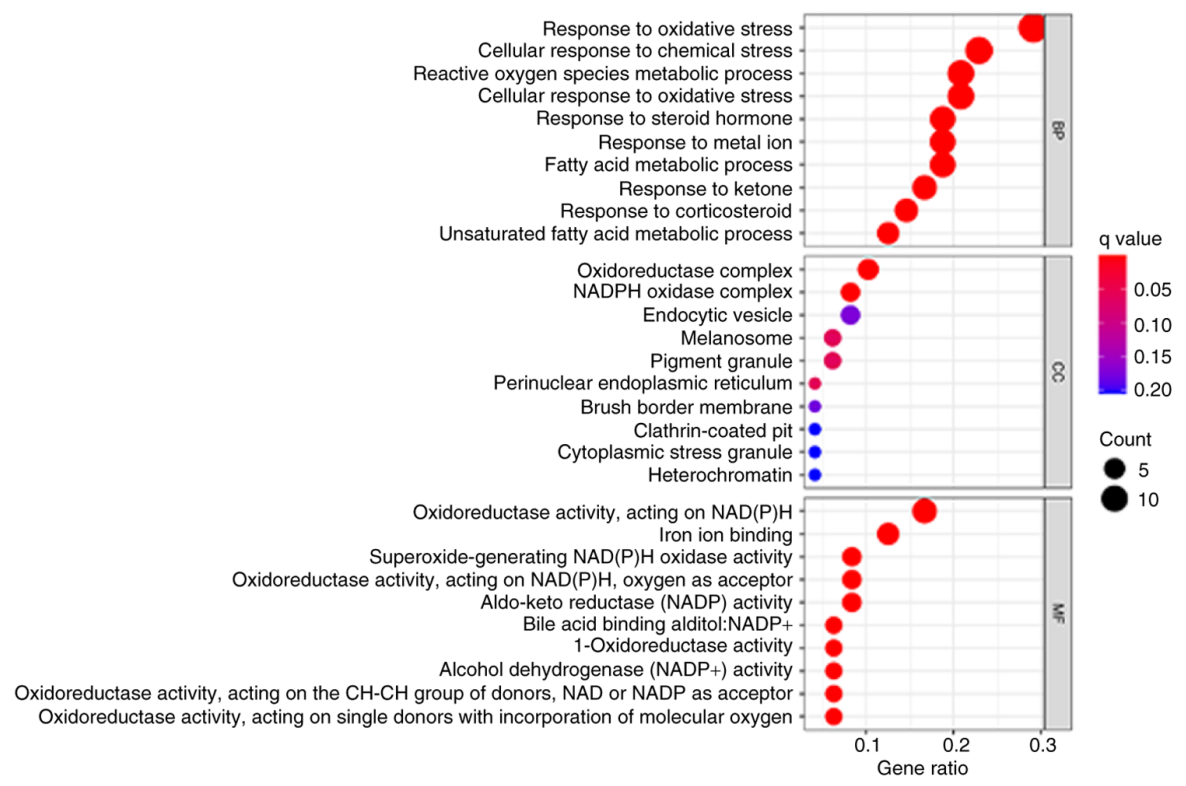

D

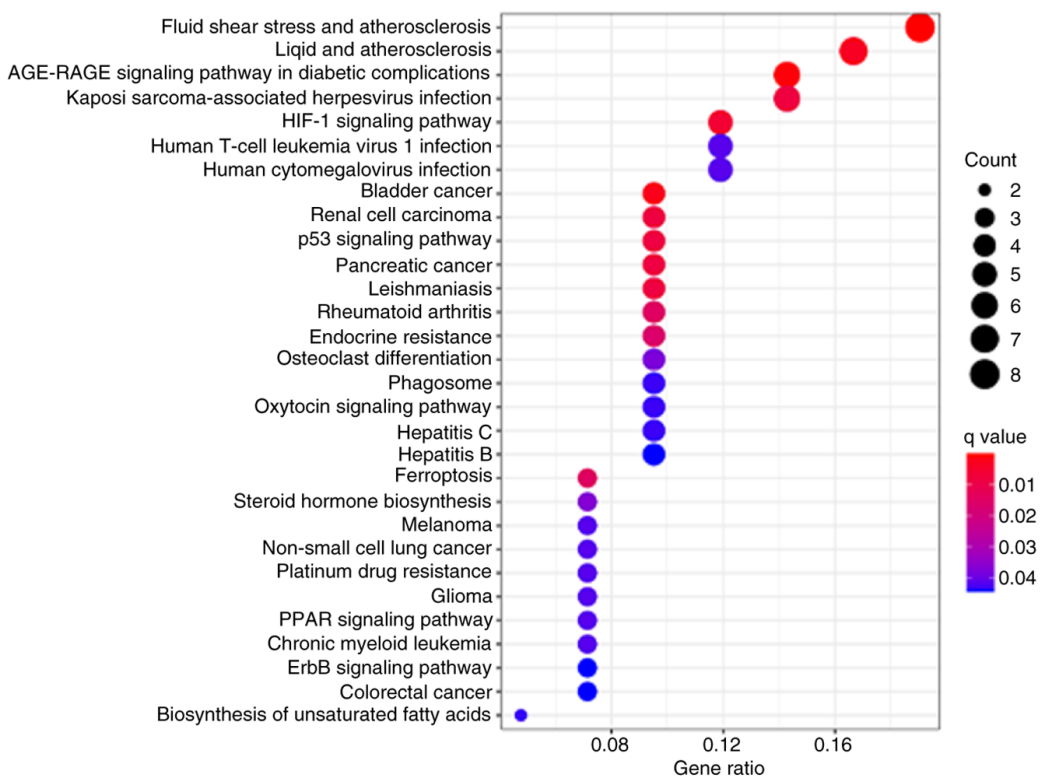

Figure 1. Expression and enrichment analyses of differentially expressed lncRNAs in individuals with stomach adenocarcinoma. (A) Heatmap of ferroptosis-related markers. (B) Heatmap of ferroptosis-related lncRNAs. (C) Bubble chart illustrating the top 10 most significant terms in the Gene Ontology functional analysis, consisting of biological process, cellular component and molecular function; the $\mathrm{x}$-axis refers to the ratio of lncRNAs abundant in the matching function. (D) Bubble chart illustrating the top 30 most significant Kyoto Encyclopedia of Genes and Genomes pathway terms of ferroptosis-related IncRNAs; the $\mathrm{x}$-axis refers to the ratio of lncRNAs abundant in the matching function. IncRNA, long noncoding RNA; N, normal tissue; T, tumor tissue. 
A

$\begin{array}{lrc}\text { A } & \text { p value } & \begin{array}{c}\text { Hazard ratio } \\ \text { C10orf55 }\end{array} \\ \text { AC007347.1 } & 0.036 & 1.422(1.023-1.976) \\ \text { MAP3K4-AS1 } & 0.018 & 1.627(1.010-2.620) \\ \text { AC026368.1 } & 0.029 & 1.040(1.019-1.226) \\ \text { AL356417.2 } & <0.001 & 2.165(1.401-3.345) \\ \text { CFAP61-AS1 } & 0.011 & 1.106(1.024-1.194) \\ \text { POLH-AS1 } & 0.030 & 0.779(0.622-0.976) \\ \text { AP002954.1 } & 0.037 & 1.270(1.015-1.590) \\ \text { AC090772.1 } & 0.012 & 2.123(1.177-3.829) \\ \text { AP005233.2 } & 0.035 & 1.043(1.003-1.085) \\ \text { LINC00449 } & 0.021 & 0.433(0.212-0.884) \\ \text { AP001363.2 } & 0.034 & 1.606(1.035-2.491) \\ \text { AC068790.7 } & 0.010 & 1.683(1.132-2.503) \\ \text { AC005165.1 } & 0.006 & 1.189(1.050-1.345) \\ \text { LINC01614 } & <0.001 & 1.127(1.055-1.204) \\ \text { LINC01711 } & <0.001 & 1.092(1.041-1.146) \\ \text { LINC01094 } & <0.001 & 1.649(1.264-2.153) \\ \text { GIHCG } & 0.041 & 1.191(1.007-1.409) \\ \text { LINC02544 } & 0.004 & 1.116(1.036-1.203) \\ \text { LINC01705 } & 0.033 & 1.093(1.007-1.186) \\ \text { AL356215.1 } & 0.026 & 1.126(1.014-1.251) \\ \text { REPIN1-AS1 } & 0.001 & 0.750(0.631-0.890) \\ \text { LASTR } & <0.001 & 1.101(1.051-1.153) \\ \text { LINC00460 } & 0.023 & 1.074(1.010-1.143) \\ \text { AC015712.1 } & 0.038 & 1.218(1.011-1.468) \\ \text { AC124067.4 } & 0.046 & 0.941(0.887-0.999) \\ \text { PVT1 } & 0.013 & 0.886(0.805-0.975) \\ & & \end{array}$

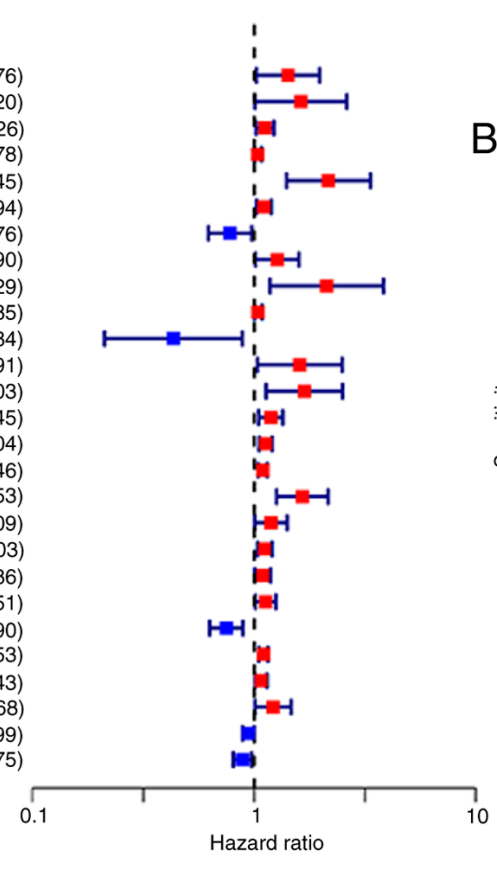

B

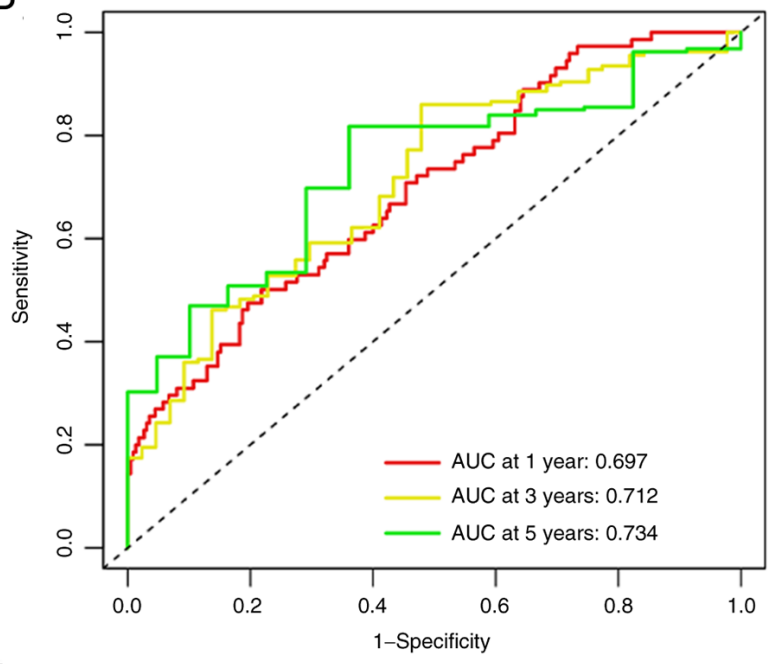

C

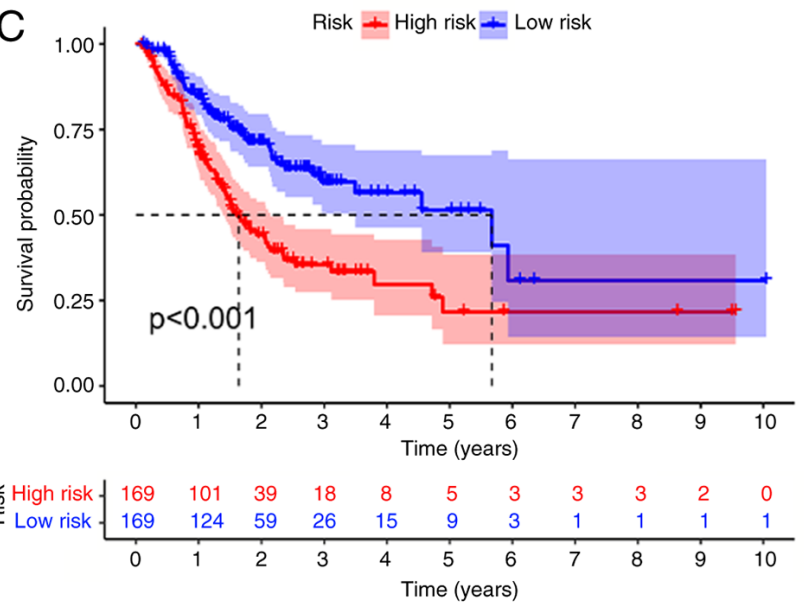

$\mathrm{E}$

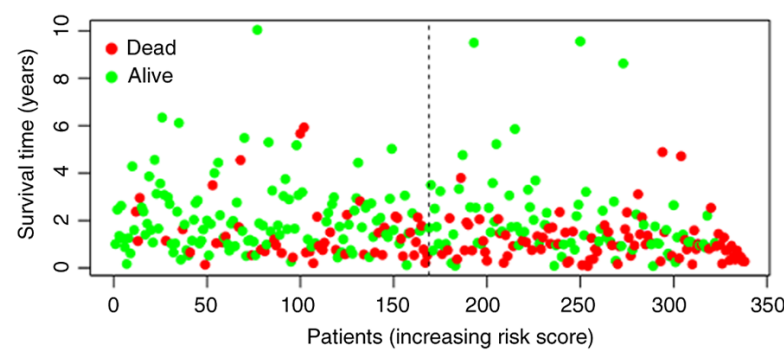

D
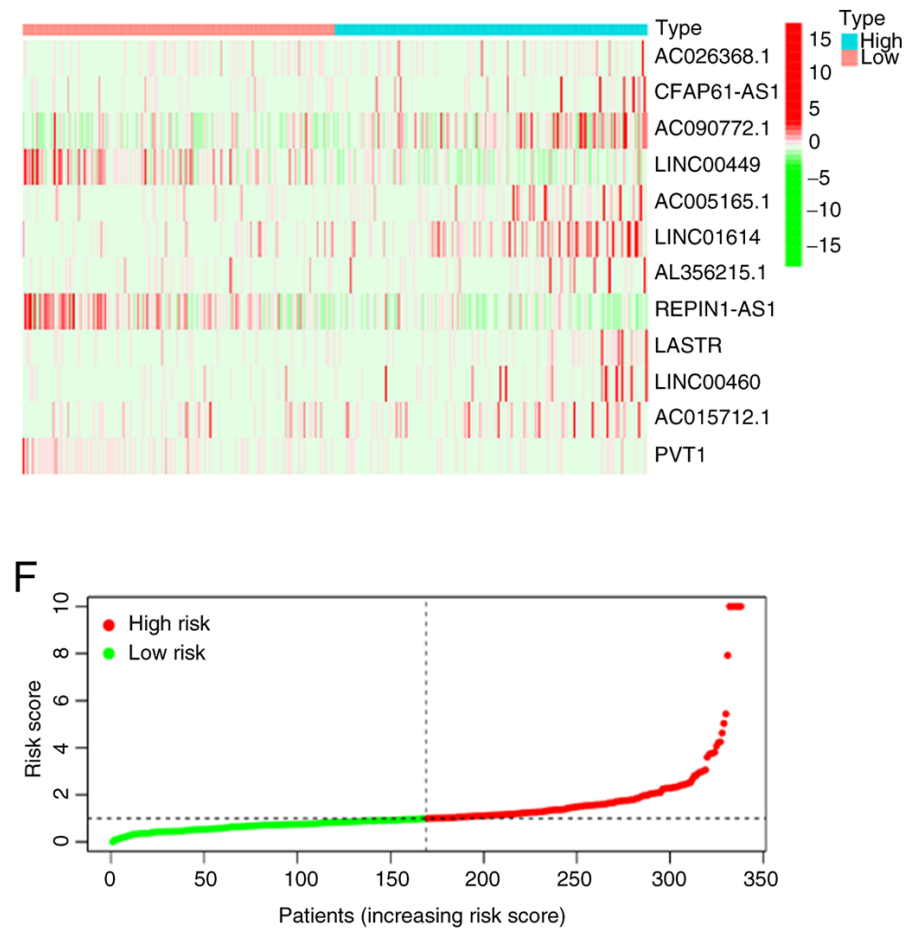

Figure 2. Establishment of 12-differentially expressed ferroptosis-related lncRNA-based OS signature. (A) Univariate Cox regression analysis established 27 ferroptosis-related lncRNAs significantly associated with OS. (B) Time-based ROC curves of the OS signature at 1, 3 and 5 years. (C) Kaplan-Meier survival plots illustrate OS differences between low-risk and high-risk groups. (D) Levels of expression of lncRNAs in the high-risk and low-risk groups according to the OS signature. (E) OS scatter plots for individuals with stomach adenocarcinoma. (F) Risk score distribution of patients according to OS signature. OS, overall survival; lncRNA, long noncoding RNA; AUC, area under the ROC curve; ROC, receiver operating characteristic.

analyses were performed. In the category biological process, the terms were related to the response to oxidative stress and the response to metal ions. The cellular component terms were associated with the production of oxidoreductase complex and NADPH oxidase complexes. In the category molecular function, the terms were related to oxidoreductase activity, iron ion binding, and superoxide-generating NAD(P)H oxidase activity
(Fig. 1C). KEGG pathway analysis indicated that primary enrichment in ferroptosis, the HIF-1 signaling pathway, the p53 signaling pathway, the PPAR signaling pathway and the ErbB signaling pathway were significant (Fig. 1D). The enrichment analyses indicated that ferroptosis-related lncRNAs are closely related to iron metabolism and may mediate certain pivotal signaling pathways in the tumorigenesis of STAD. 
A

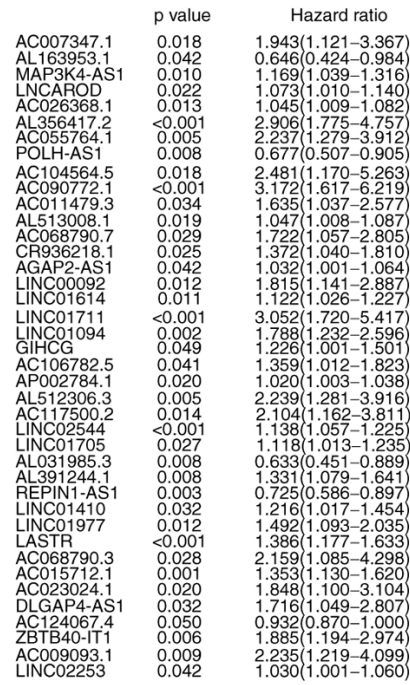

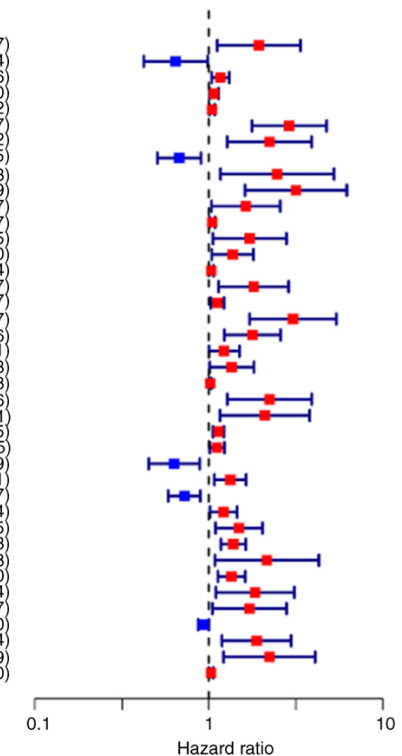

C

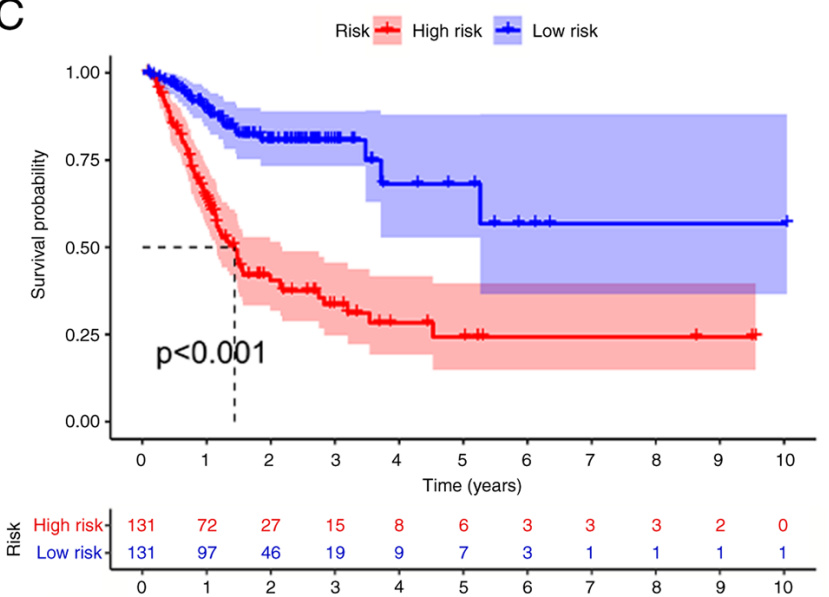

E

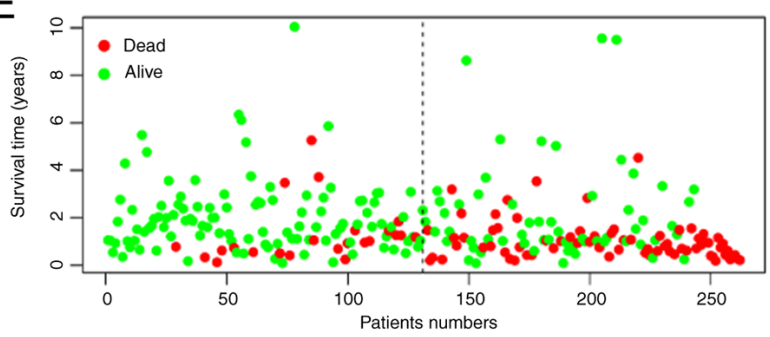

B

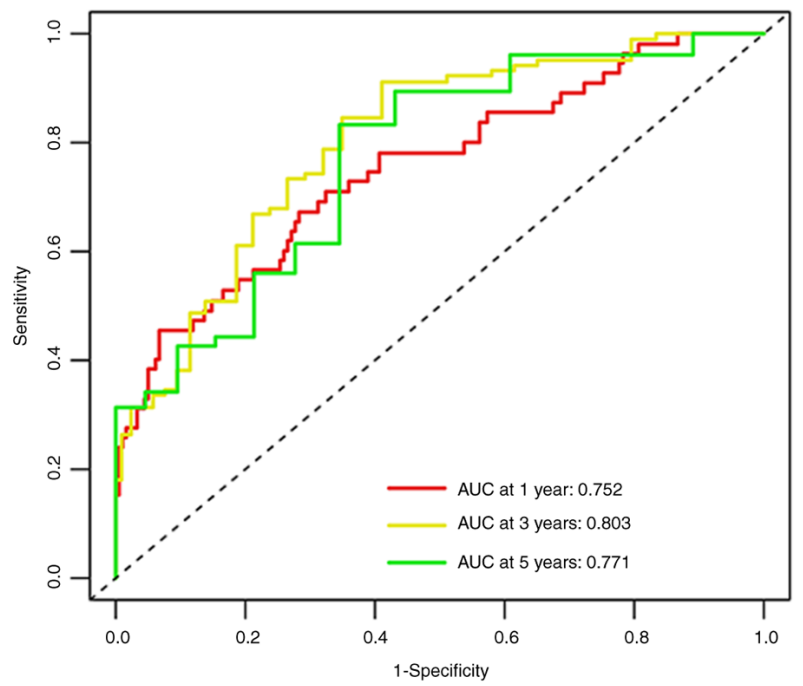

D

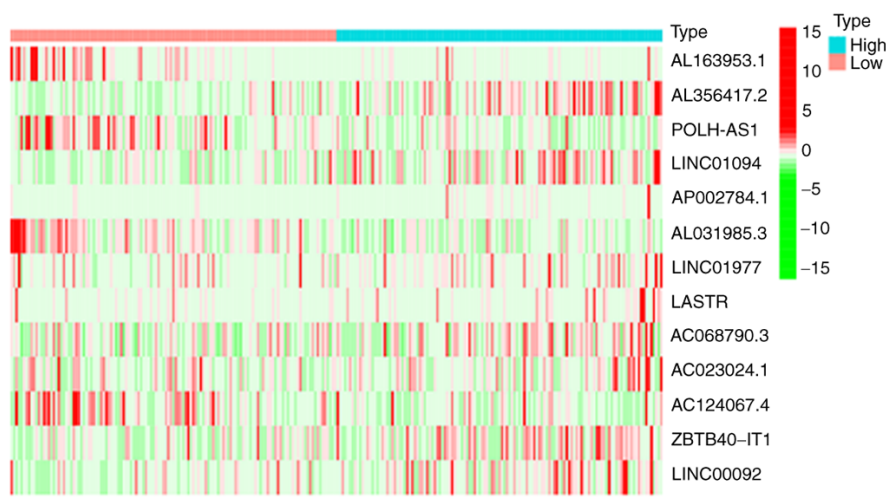

$\mathrm{F}$

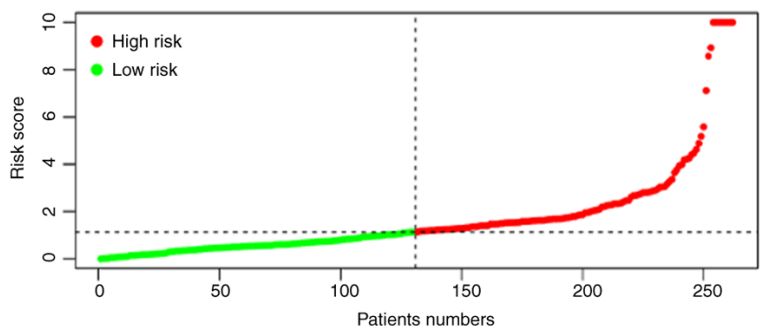

Figure 3. Establishment of 13-differentially expressed ferroptosis-related lncRNA-based PFS signature. (A) Univariate Cox regression uncovered 40 ferroptosis-related lncRNAs that were significantly associated with PFS. (B) Time-based ROC curves of the PFS signature at 1, 3 and 5 years. (C) Kaplan-Meier survival plots illustrate PFS differences between the low-risk and high-risk groups. (D) Levels of lncRNA expression in the high-risk and low-risk groups according to the PFS signature. (E) PFS scatter plots for individuals with stomach adenocarcinoma. (F) Risk score distribution of patients according to PFS signature. PFS, progression-free survival; lncRNA, long noncoding RNA; AUC, area under the ROC curve; ROC, receiver operating characteristic.

Prognostic value of ferroptosis-related lncRNAs in STAD. The lack of reliable markers for early tumor diagnosis is still one of the critical factors in the dismal prognosis of individuals with advanced STAD. Recent studies have revealed that ferroptosis-related lncRNAs may act as prognostic targets in diverse cancers $(14,15)$. Thus, the possible predictive value of ferroptosis-related lncRNAs in individuals with STAD was further confirmed (Figs. 2 and 3). Univariate Cox analysis uncovered 27 and 40 ferroptosis-related lncRNAs that were significantly related to $\mathrm{OS}$ and $\mathrm{PFS}$, respectively (Figs. 2A and 3A). Subsequently, multivariate Cox analysis was performed and a total of 25 ferroptosis-related lncRNAs were employed to produce two predictive signatures, consisting of 12 ferroptosis-related lncRNAs for the OS signature and 13 ferroptosis-related lncRNAs for the PFS signature. The formula for the OS signature was as follows: Risk score $=0.03525 \times$ AC026368.1 + 0.09945 $\mathrm{x}$ CFAP61-AS1 + 1.07809 x AC090772.1-0.62697 x LINC00449-0.12656 x AC005165.1 + 0.14984 x LINC01614 + 0.11706 x AL356215.1-0.17220 x REPIN1-AS1 + 0.05905 x LASTR+0.07243xLINC00460+0.21417x AC015712.1-0.13778 $\mathrm{xPVT1}$.ThePFS signature was as follows: Risk score $=-0.47559 \mathrm{x}$ 
A

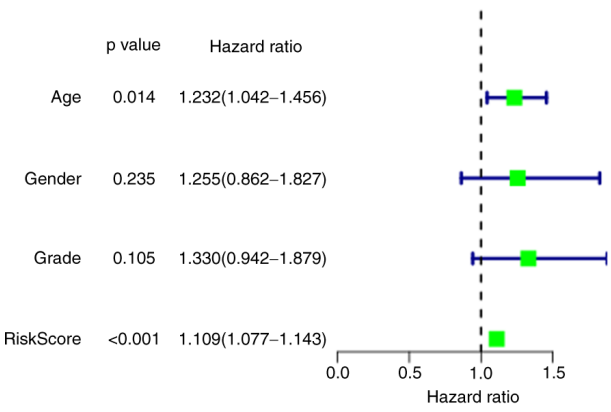

C

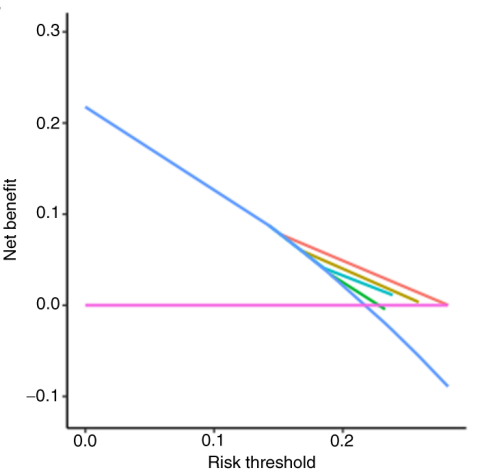

$\mathrm{E}$

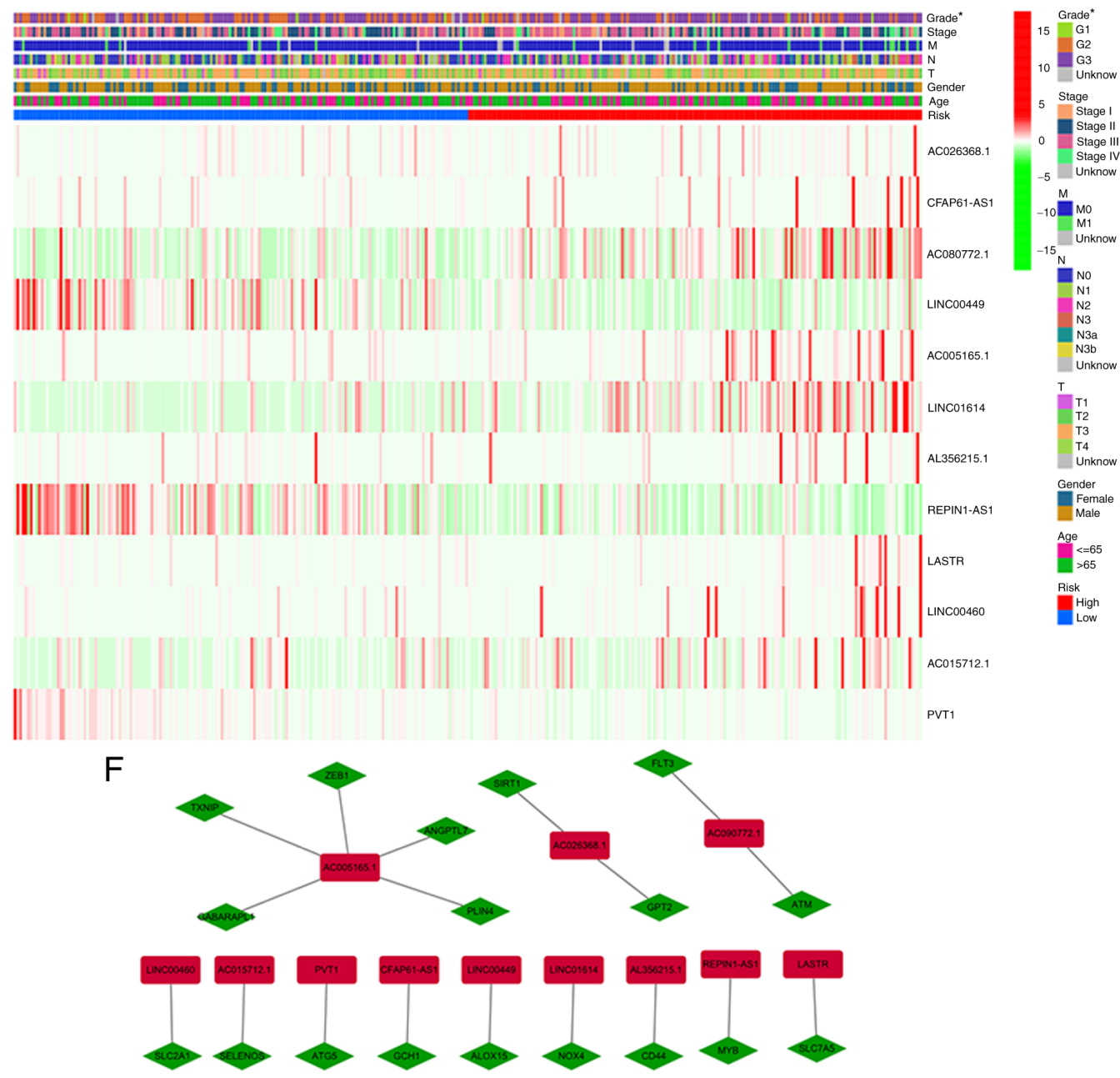

Figure 4. Development of nomogram combining the DEFRL-based signature with independent predictive clinical variables to estimate OS in individuals with stomach adenocarcinoma. (A) Univariate regression of DEFRL-based prognostic signature along with clinical factors. (B) Multivariate regression of the significant characteristics in the univariate regression. (C) Decision Curve Analysis of the risk factors with OS. (D) Nomogram of OS integrating the OS signature with the two clinical variables of patients. (E) Heatmap illustrating ferroptosis-related IncRNAs by OS signature and clinicopathological manifestations. (F) Regulatory network of ferroptosis-related markers and OS-linked ferroptosis-related lncRNAs. ${ }^{*} \mathrm{P}<0.05,{ }^{* * *} \mathrm{P}<0.01$ and ${ }^{* * * *} \mathrm{P}<0.001$. OS, overall survival; lncRNA, long noncoding RNA; DEFRL, differentially expressed ferroptosis-related lncRNAs; Pr, probability. 

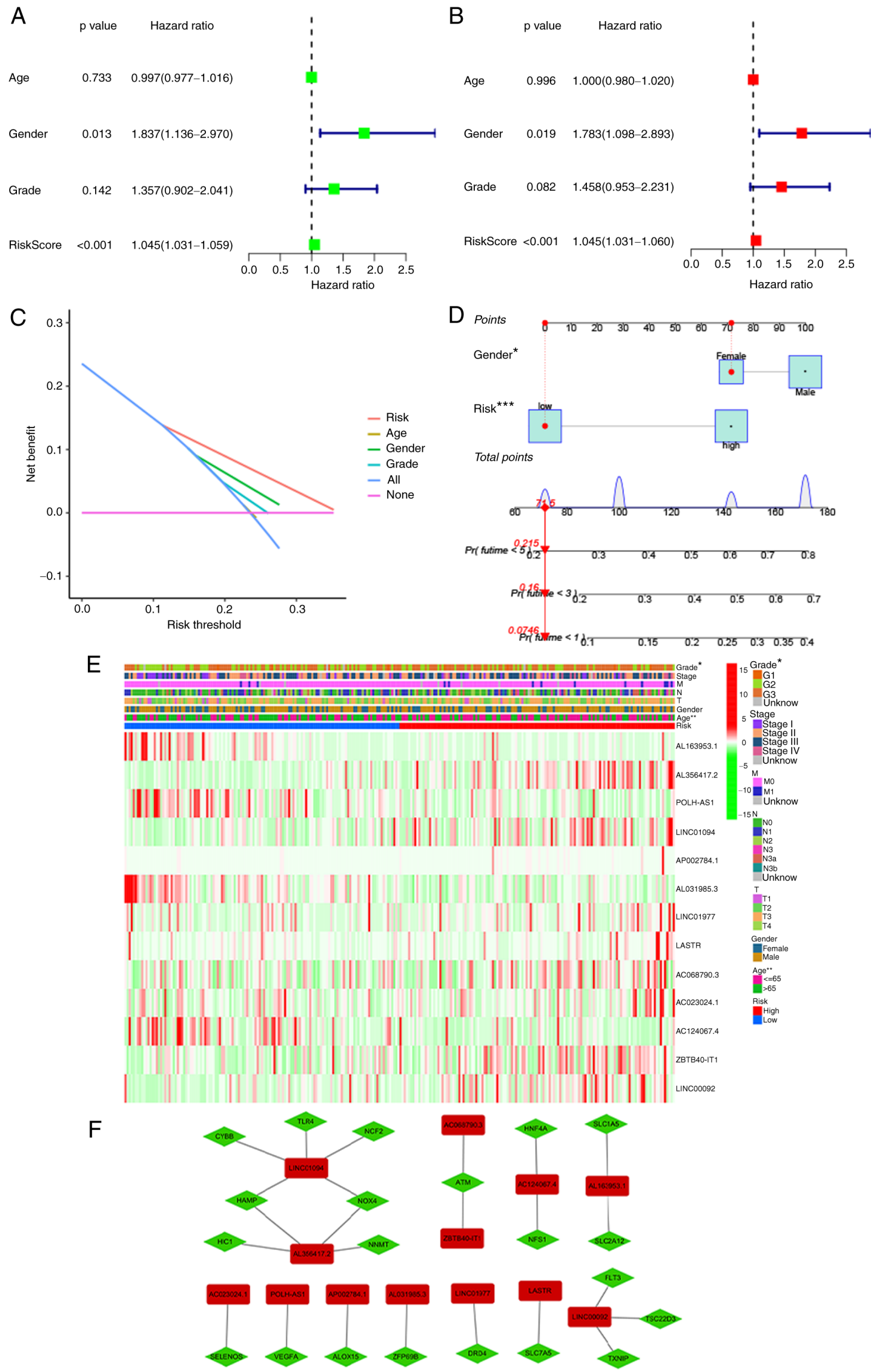

Figure 5. Development of nomogram combining DEFR-based signature with independent predictive clinical variables to predict PFS in individuals with stomach adenocarcinoma. (A) Univariate regression of the DEFRL-based predictive signature along with clinical factors. (B) Multivariate regression of the significant characteristics in the univariate Cox analyses. (C) DCA of the risk factors with PFS. (D) Nomogram of PFS integrating the PFS signature with the two clinical variables of patients. (E) Heatmap illustrating the ferroptosis-related lncRNA PFS signature and clinicopathological manifestations. (F) Modulatory network of ferroptosis-related markers and PFS-linked ferroptosis-related lncRNAs. ${ }^{*} \mathrm{P}<0.05,{ }^{* *} \mathrm{P}<0.01$, and ${ }^{* * *} \mathrm{P}<0.001$. PFS, progression-free survival; lncRNA, long noncoding RNA; DEFRL, differentially expressed ferroptosis-related lncRNAs; Pr, probability. 
A

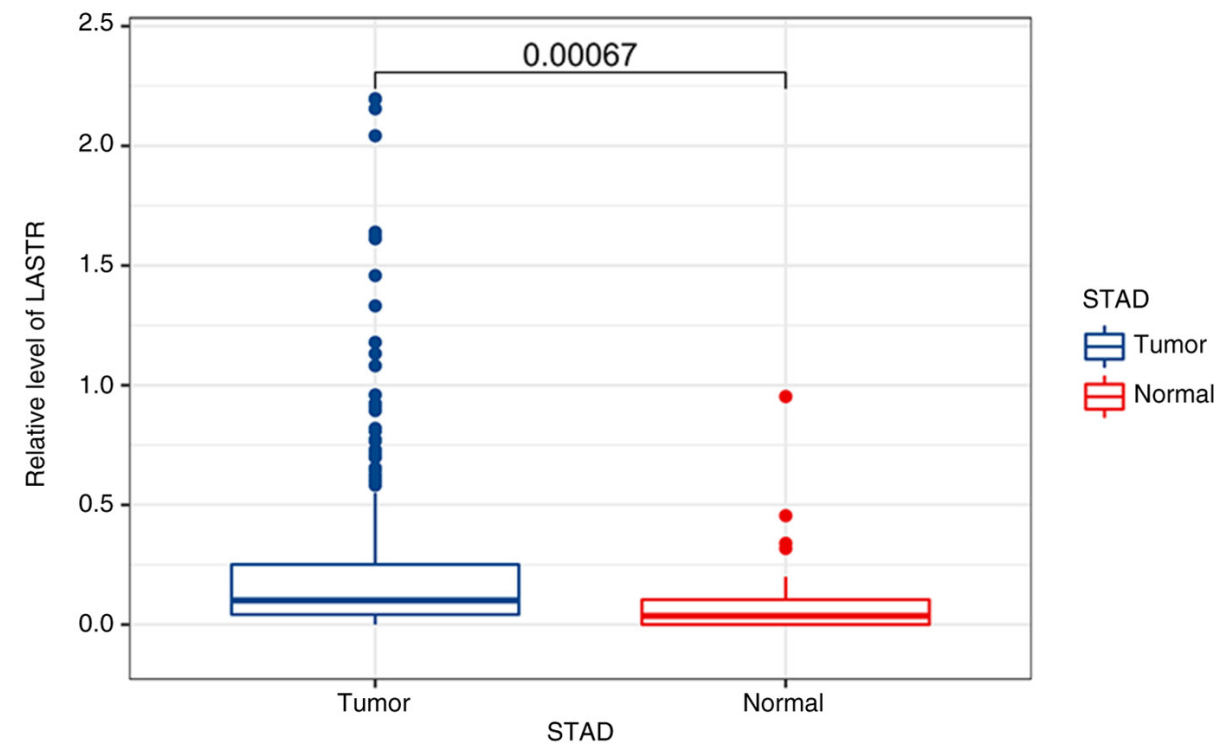

B

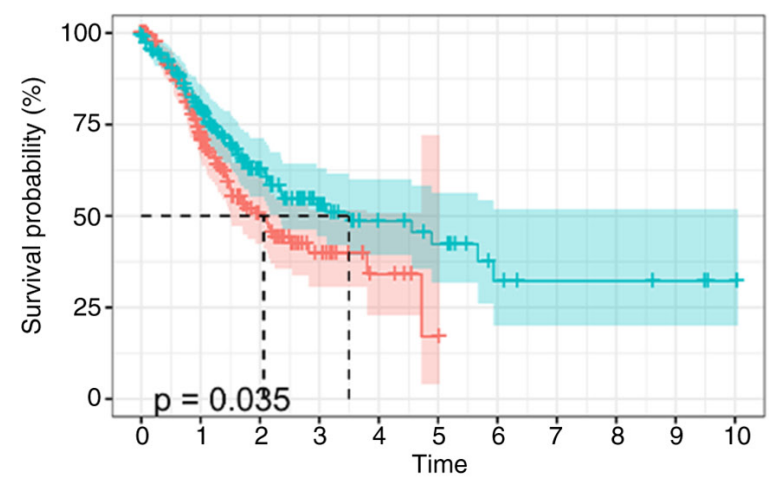

Number at risk
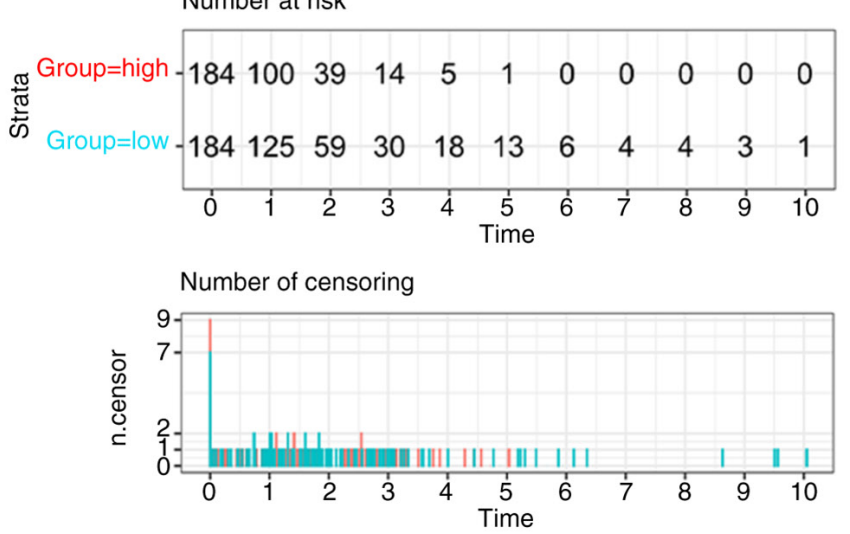

C Strata + Group=high + Group=low

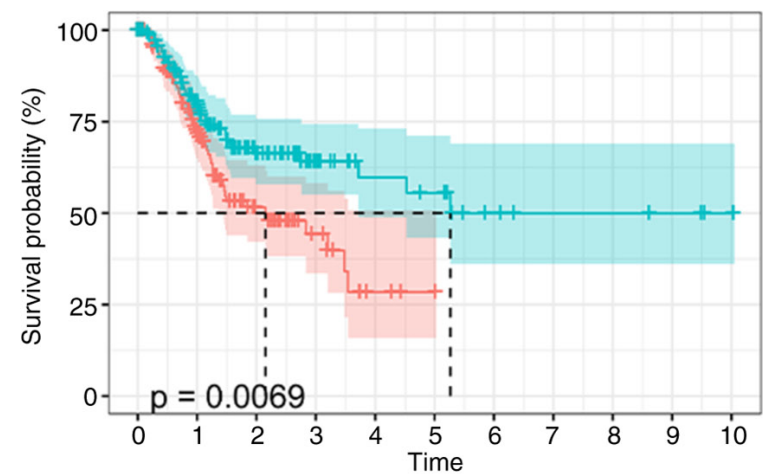

Number at risk
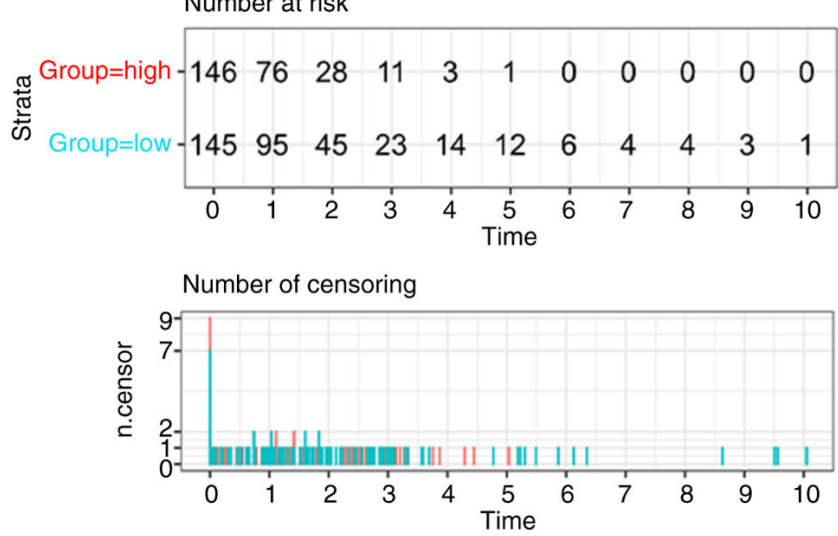

Figure 6. High expression of LASTR predicts poor prognosis in individuals with stomach adenocarcinoma. (A) LASTR expression in gastric cancer tissues and neighboring non-malignant tissues from The Cancer Genome Atlas database. (B) Kaplan-Meier survival plots illustrating the differences in OS between low-level and high-level LASTR. (C) Kaplan-Meier survival plots illustrating PFS differences between low-level and high-level LASTR. STAD, stomach adenocarcinoma; LASTR, IncRNA associated with spliceosome associated factor 3, U4/U6 recycling protein regulation of splicing.

AL163953.1 + 0.972225 x AL356417.2-0.28607 xPOLH-AS1 + 0.377634 x LINC01094 + 0.034465 x AP002784.1-0.75762 x AL031985.3 + 0.532136 x LINC01977 + 0.300628 x LASTR + $0.838305 \times$ AC068790.3 + 0.70416 x AC023024.1-0.0644 x AC124067.4 + $0.578635 \times$ ZBTB40-IT1 + $0.434186 x$ LINC00092. The time-based ROC curves illustrated that the AUCs of the OS signature for estimating 1-, 3- and 5-year OS were 0.697, 0.712 and 0.734 , respectively (Fig. 2B). Subsequently, based on the median risk score, patients were divided into high- and low-risk groups. The K-M plots illustrated that the high-risk group had a dismal OS in comparison with the low-risk group (Fig. 2C). The AUCs of the signature for estimating 1-, 3- and 5-year PFS were 0.752, 0.803 and 0.771, respectively (Fig. 3B). Similar to the situation for the OS signature, those patients with STAD with higher risk scores had unfavorable PFS (Fig. 3C). These data illustrated that the OS and PFS signature were valuable tools for estimating the prognosis of individuals with STAD. To more clearly illustrate 
A

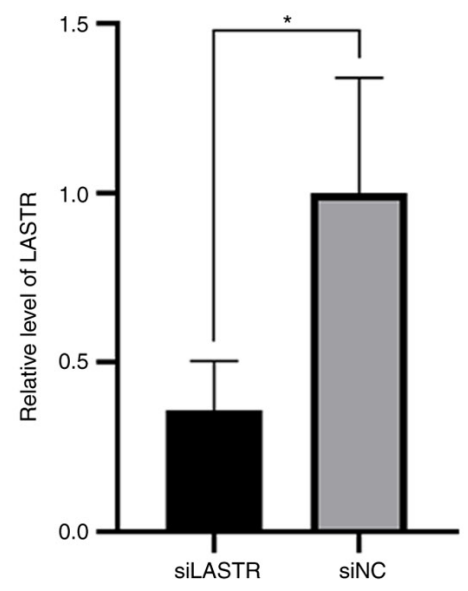

C

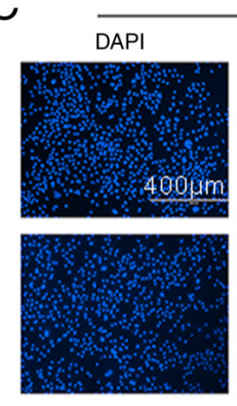

AGS

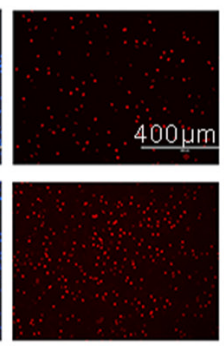

$\mathrm{E}$

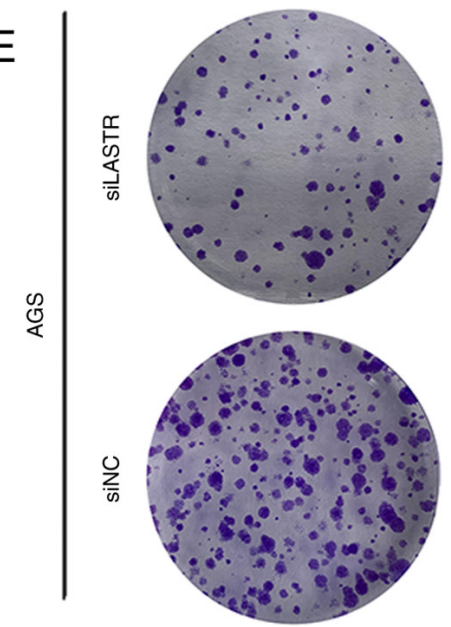

$\mathrm{B}$

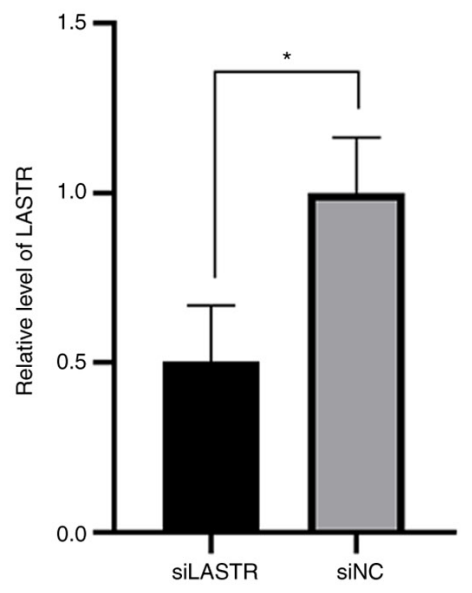

D

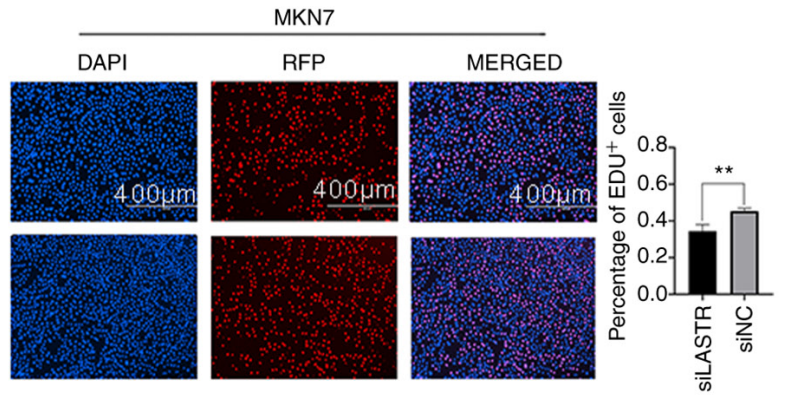

$\mathrm{F}$

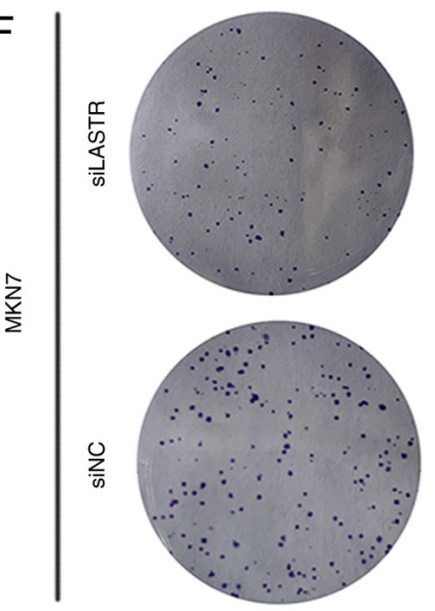

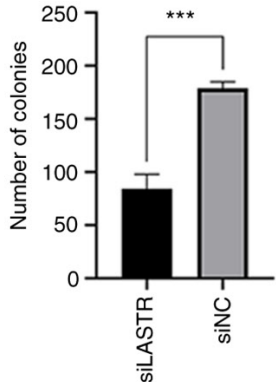

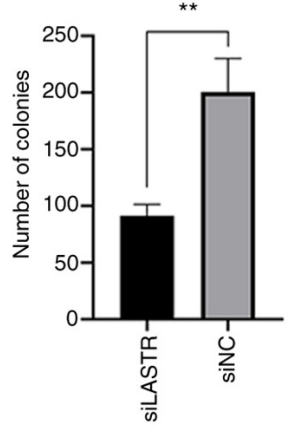

Figure 7. Impact of LASTR knockdown on the growth of gastric cancer cells. (A and B) LASTR RNA expression in (A) AGS or (B) MKN7 cells transfected with siLASTR and siNC was confirmed by reverse transcription-quantitative PCR. (C and D) Comparison of the proliferative potential between the siLASTR and siNC groups of (C) AGS or (D) MKN7 cells by an EdU incorporation assay. (E and F) Comparison of the clonogenic potential between the siLASTR and siNC groups of (E) AGS or (F) MKN7 cells by a colony formation assay. ${ }^{*} \mathrm{P}<0.05,{ }^{* *} \mathrm{P}<0.01,{ }^{* * *} \mathrm{P}<0.001$ and ${ }^{* * * * *} \mathrm{P}<0.0001$. LASTR, lncRNA associated with spliceosome associated factor 3, U4/U6 recycling protein regulation of splicing; siLASTR, small interfering RNA targeting LASTR; NC, negative control; RFP, red fluorescent protein; EdU, 5-ethynyl-2'-deoxyuridine.

differences in the prognosis and expression trends of lncRNAs, heatmaps were then constructed (Figs. 2D and 3D), as well as survival status plots (Figs. 2E and 3E) and risk score plots (Figs. $2 \mathrm{~F}$ and $3 \mathrm{~F}$ ). All of the above results indicated that the signatures based on DEFRLs are able to effectively assess the prognosis of patients with STAD.

Creation of nomograms on the basis of the DEFRL signatures and clinical parameters. To enhance the clinical utility of the prognostic signatures determined in the present study, two comprehensive signatures were constructed based on independent clinical parameters (Figs. 4 and 5). First, univariate and multivariate Cox analyses were performed to explore independent predictive variables of OS along with PFS. Risk score and age were identified as independent OS-related variables (Fig. 4A and B). Two independent variables, namely the risk score and sex, were identified as PFS-related variables (Fig. 5A and B). Clinical factors of patients with STAD that correlated with Decision Curve Analysis parameters were screened (Figs. 4C and 5C). Furthermore, on the basis of the independent predictive variables, two new nomograms were created to estimate 
A
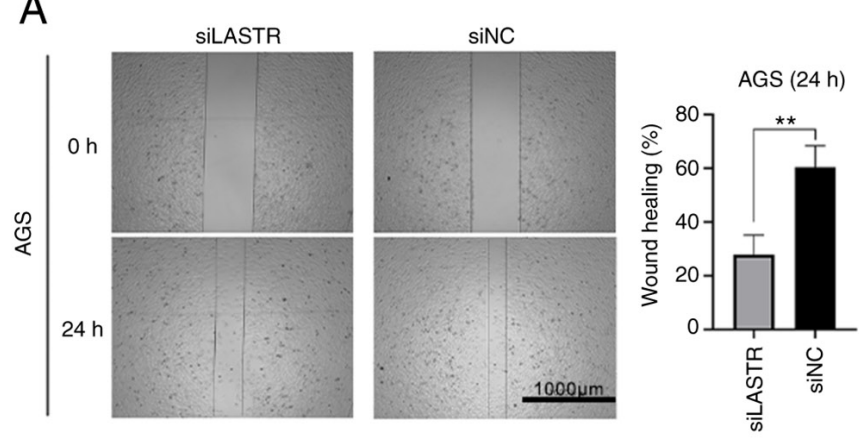

C
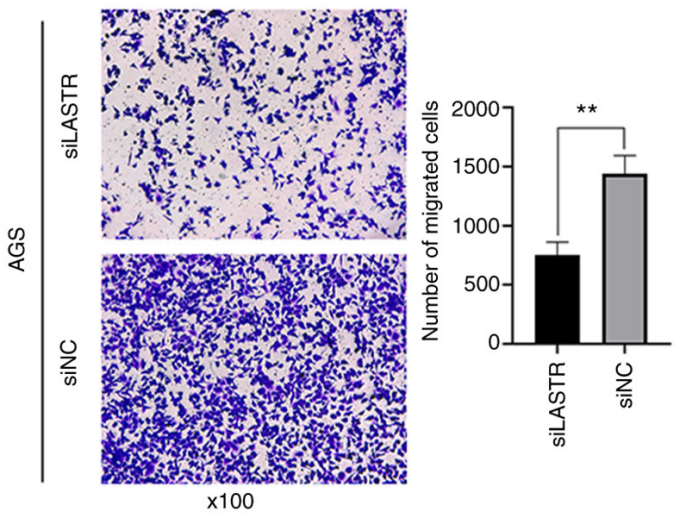

E
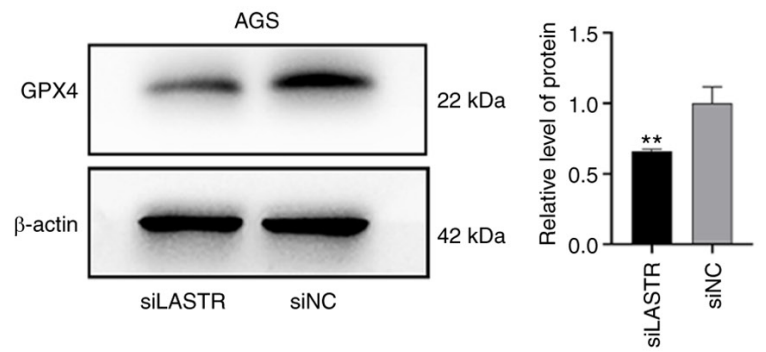

B
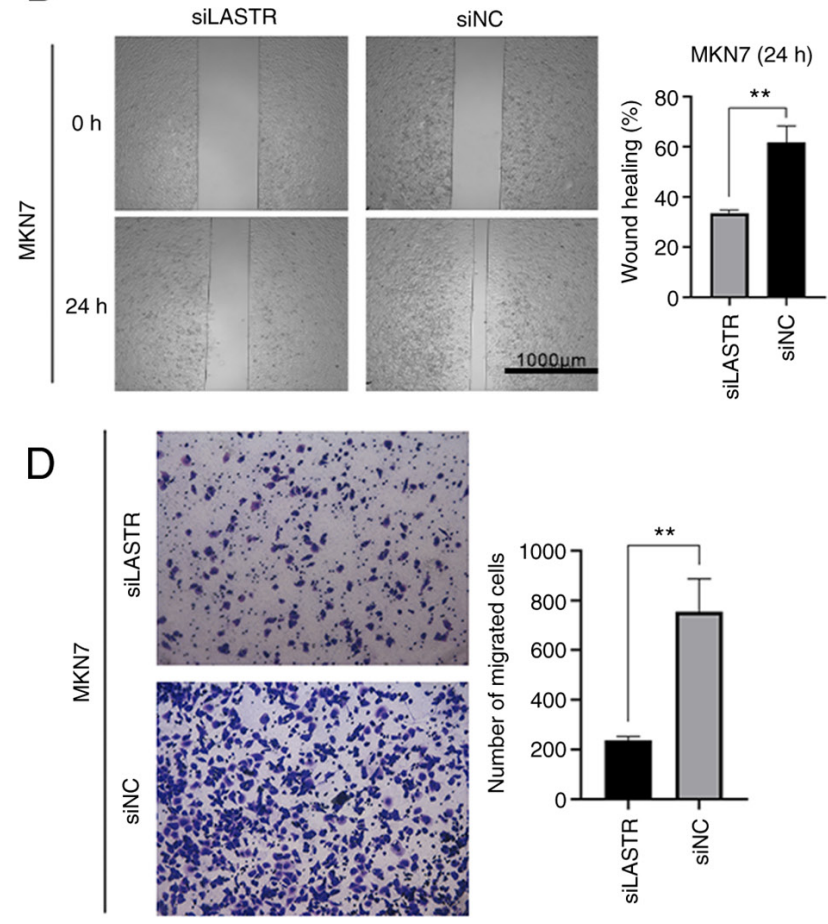

$\times 100$

$\mathrm{F}$
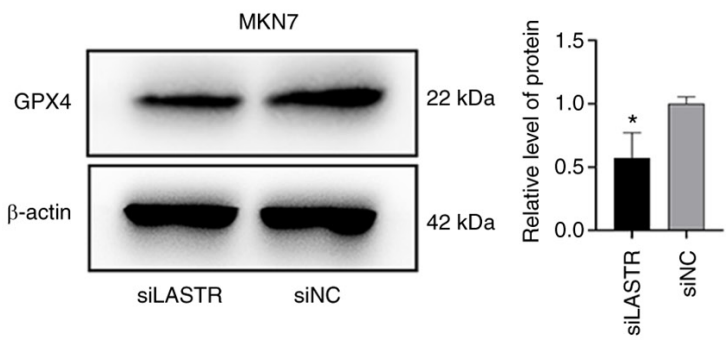

Figure 8. Impact of LASTR knockdown on the migration and the ferroptosis of gastric cancer cells. (A and B) Wound-healing assays comparing the migration distance between the siLASTR and siNC groups of (A) AGS or (B) MKN7 cells. (C and D) Transwell assay comparing the number of migrated cells between the siLASTR and siNC groups of (C) AGS or (D) MKN7 cells. (E and F) Comparison of the protein expression of GPX4 between the siLASTR and siNC groups of (E) AGS or (E) MKN7 cells by western blot assay. ${ }^{*} \mathrm{P}<0.05$ and ${ }^{* *} \mathrm{P}<0.01$. LASTR, lncRNA associated with spliceosome associated factor 3 , U4/U6 recycling protein regulation of splicing; siLASTR, small interfering RNA targeting LASTR; NC, negative control; GPX4, phospholipid hydroperoxide glutathione peroxidase 4 .

OS and PFS (Figs. 4D and 5D). The C-indices were 0.674 (95\% CI, 0.625-0.723) and 0.664 (95\% CI, 0.6052-0.7228) for the OS and PFS nomograms. These data illustrated that two nomograms may be adapted to precisely estimate the prognosis of individuals with STAD. Heatmaps for the associations of ferroptosis-related lncRNA prognostic signatures with clinicopathological manifestations were also generated (Figs. 4E and 5E). Furthermore, the association between IncRNAs and mRNAs for the OS and PFS signatures is displayed in Figs. 4F and 5F, revealing a complex regulatory relationship between them.

Roles of LASTR in GC. One overlapping ferroptosis-related lncRNA among those identified by multivariate regression of OS and PFS signatures, namely LASTR, was indicated to be the most critical factor in the prognostic models for STAD. It was reported that LASTR was able to foster the fitness of cancer cells via modulating the activity of the U4/U6 recycling factor SART3 (16). The specific roles of
LASTR in GC cells were then explored. The expression of LASTR in GC tissues and neighboring non-malignant tissues from the TCGA data resource is provided in Fig. 6A. K-M survival curves illustrated that a low level of LASTR was significantly associated with favorable OS and PFS (Fig. 6B and C). Next, siLASTR was used to infect two cell lines (AGS and MKN7) and the knockdown efficiency was confirmed via RT-qPCR (Fig. 7A and B). Subsequently, an EdU incorporation assay (Fig. 7C and D) and colony formation assay (Fig. 7E and F) were performed on the two GC cell lines to measure changes in proliferative capacity, and a wound-healing assay (Fig. 8A and B) and Transwell migration assay (Fig. 8C and D) were carried out to assess changes in migration capacity. The results suggested that the cell proliferation and migration abilities were decreased following LASTR knockdown. Western blot analysis was used to compare the expression of the ferroptosis marker GPX4 between the knockdown group and control group. Compared with the control group, the 
level of GPX4 was significantly decreased in the knockout group (Fig. 8E and F). The results indicated that knockdown of LASTR repressed cell growth and migration and may trigger ferroptosis in GC.

\section{Discussion}

Despite advances in detection approaches and medical standards, the five-year survival rate of patients with STAD remains low (17). One of the main reasons is the lack of sufficiently specific and sensitive biomarkers for early diagnosis (18). It has been indicated that ferroptosis is involved in proliferation, migration, drug resistance and other biological behaviors of STAD (19-23). LncRNAs are pivotal regulators of ferroptosis, having different biological roles in various cancer types (7-9,24-27). However, an effective predictive tool featuring ferroptosis-related lncRNAs for patients with STAD is still lacking. In the present study, 12 and 13 ferroptosis-related lncRNAs were identified and used to produce signatures to predict OS and PFS, respectively, in patients with STAD. First, 382 ferroptosis-related markers were determined from the TCGA dataset and 503 lncRNAs were identified as candidate predictive biomarkers. Furthermore, and GO and KEGG analyses uncovered the prospective mechanisms of these lncRNAs. Of note, an OS predictive signature was constructed based on 12 key lncRNAs and a PFS predictive signature consisting of 13 key lncRNAs. According to the risk scores, STAD subjects were categorized into high-risk and low-risk groups. The differences in OS and PFS between the high-risk and low-risk groups were statistically significant. The reliability of these two signatures was further supported by the prediction ability of the ROC curves of the two signatures. In addition, two comprehensive nomograms integrating the IncRNA-related prognostic signatures and clinical features were established to enhance clinical utility. These nomograms allow clinicians to evaluate the OS and PFS for each patient with STAD by inputting the score for each parameter.

The present study also indicated that oxidative stress and tumor-associated signaling pathways, such as the HIF-1 signaling pathway, the p53 signaling pathway, the PPAR signaling pathway and the ErbB signaling pathway, were significantly enriched through GO and KEGG functional enrichment analyses. It was previously reported that ferroptosis induced by oxidative stress is associated with various diseases, such as Alzheimer's disease (28), intervertebral disc degeneration (29) and cancer (30). Ni et al (31) demonstrated that targeting HIF-1 $\alpha$ is able to induce osteoclast ferroptosis to treat osteoporosis. Certain studies have indicated that p53 is able to regulate ferroptosis and mediate certain diseases $(32,33)$. The above studies have proved the regulatory relationship between signaling pathways and ferroptosis in different disease types, confirming the reliability of the signatures constructed in the present study.

A total of 12 lncRNAs (AC026368.1, CFAP61-AS1, AC090772.1, LINC00449, AC005165.1, LINC01614, AL356215.1, REPIN1-AS1, LASTR, LINC00460, AC015712.1 and PVT1) were included in the OS signature and 13 lncRNAs (AL163953.1, AL356417.2, POLH-AS1, LINC01094, AP002784.1, AL031985.3, LINC01977,
LASTR, AC068790.3, AC023024.1, AC124067.4, ZBTB40-IT1 and LINC00092) in the PFS signature. LINC00449 (34), LINC01614 (35-39), LINC00460 (40-44), PVT1 (24,26,45-49), LINC01094 (50-55), LINC01977 (56), ZBTB40-IT1 (57) and LINC00092 (58) were previously reported to regulate the biological behavior or serve as prognostic tumor biomarkers in various cancer types. Except for these lncRNAs mentioned above, the other lncRNAs included in the signatures have remained largely unexplored, which will be the focus of future studies by our group. Among the OS and PFS signatures, LASTR was the only lncRNA included in both signatures, which may thus have a relatively greater prognostic value in patients with STAD. LASTR (LINC02657) was originally named as it was indicated to be associated with SART3 regulation of splicing. It was previously reported that LASTR was upregulated in triple-negative breast cancer with hypoxia and affected the adaptability of tumor cells (16). Apart from that, there is virtually no information about it in the literature. Thus, the biological roles of LASTR in GC were further explored in the present study. Cell experiments, omics experiments and bioinformatics analysis were performed to confirm that LASTR has a role in enhancing GC progression. When verifying the association between LASTR and ferroptosis, due to limitations of experimental technology and the experimental environment, only the changes in the ferroptosis marker GPX4 protein after LASTR was knocked down were examined to determine their regulatory relationship. As this analysis was not very rigorous, this matter will be explored in advanced research settings in the future.

Ferroptosis has become a hot topic in research in recent years and provides a novel mechanism for cancer treatment. There are still numerous unknown areas in the relation between ferroptosis and IncRNAs worth exploring. In the present study, 12 and 13 ferroptosis-related lncRNAs were identified and included in an OS signature and PFS signature for STAD, respectively, and experimental verification of the roles of LASTR was performed.

In conclusion, in the present study, novel ferroptosis-related biomarkers were identified for STAD prognosis. One of the markers, LASTR, was experimentally verified as a cancer-promoting factor and to be associated with ferroptosis. This study provided a novel approach for the treatment of cancer and predict the survival of patients with STAD.

\section{Acknowledgements}

Not applicable.

\section{Funding}

This study was supported by grants from the Beijing Xisike Clinical Oncology Research Foundation (grant no. Y-BMS2019-038), Wu Jieping Medical Foundation (grant no. 320.6750.19088-29), Shandong Medical and Health Technology Development Foundation (grant no. 202003030451) and Qingdao Municipal People's Livelihood Science and Technology Foundation (grant no. 17-3-3-34-nsh). 


\section{Availability of data and materials}

The datasets used and/or analyzed during the current study are available from the corresponding author on reasonable request.

\section{Authors' contributions}

GW, LS and SW analyzed the data. GW and JG wrote and reviewed the manuscript. WQiu, WQi and JG contributed to the design of the study. GW, RX and WL performed the experiments. All authors contributed to the article and read and approved the final version. All authors confirm the authenticity of the raw data.

\section{Ethics approval and consent to participate}

Not applicable.

\section{Patient consent for publication}

Not applicable.

\section{Competing interests}

The authors declare that they have no competing interests.

\section{References}

1. Bray F, Ferlay J, Soerjomataram I, Siegel RL, Torre LA and Jemal A: Global cancer statistics 2018: GLOBOCAN estimates of incidence and mortality worldwide for 36 cancers in 185 countries. CA Cancer J Clin 68: 394-424, 2018.

2. Chen W, Zheng R, Baade PD, Zhang S, Zeng H, Bray F, Jemal A, Yu XQ and He J: Cancer statistics in China, 2015. CA Cancer J Clin 66: 115-132, 2016.

3. Van Cutsem E, Sagaert X, Topal B, Haustermans K and Prenen H: Gastric cancer. Lancet 388: 2654-2664, 2016.

4. Tang D, Kang R, Berghe TV, Vandenabeele P and Kroemer G: The molecular machinery of regulated cell death. Cell Res 29: 347-364, 2019

5. Stockwell BR, Friedmann Angeli JP, Bayir H, Bush AI, Conrad M, Dixon SJ, Fulda S, Gascón S, Hatzios SK, Kagan VE, et al: Ferroptosis: A regulated cell death nexus linking metabolism, redox biology, and disease. Cell 171: 273-285, 2017

6. Hassannia B, Vandenabeele P and Vanden Berghe T: Targeting ferroptosis to Iron out cancer. Cancer Cell 35: 830-849, 2019.

7. Zhang Y, Guo S, Wang S, Li X, Hou D, Li H, Wang L, Xu Y, Ma B, Wang $\mathrm{H}$ and Jiang X: LncRNA OIP5-AS1 inhibits ferroptosis in prostate cancer with long-term cadmium exposure through miR-128-3p/SLC7A11 signaling. Ecotoxicol Environ Saf 220: 112376, 2021.

8. Ma Q, Dai X, Lu W, Qu X, Liu N and Zhu C: Silencing long non-coding RNA MEG8 inhibits the proliferation and induces the ferroptosis of hemangioma endothelial cells by regulating miR-497-5p/NOTCH2 axis. Biochem Biophys Res Commun 556: $72-78,2021$.

9. Wang Z, Chen X, Liu N, Shi Y, Liu Y, Ouyang L, Tam S, Xiao D, Liu S, Wen $\mathrm{F}$ and Tao Y: A nuclear long non-coding RNA LINC00618 accelerates ferroptosis in a manner dependent upon apoptosis. Mol Ther 29: 263-274, 2021.

10. Gao J, Aksoy BA, Dogrusoz U, Dresdner G, Gross B, Sumer SO, Sun Y, Jacobsen A, Sinha R, Larsson E, et al: Integrative analysis of complex cancer genomics and clinical profiles using the cBioPortal. Sci Signal 6: pl1, 2013.

11. Zhou N and Bao J: FerrDb: A manually curated resource for regulators and markers of ferroptosis and ferroptosis-disease associations. Database (Oxford) 2020: baaa021, 2020

12. Yu G, Wang LG, Han Y and He QY: clusterProfiler: An R package for comparing biological themes among gene clusters. OMICS 16: 284-287, 2012 .
13. Chew WL, Tabebordbar M, Cheng JK, Mali P, Wu EY, $\mathrm{Ng} \mathrm{AH}$, Zhu K, Wagers AJ and Church GM: A multifunctional AAV-CRISPR-Cas9 and its host response. Nat Methods 13: 868-874, 2016

14. Cai HJ, Zhuang ZC, Wu Y, Zhang YY, Liu X, Zhuang JF, Yang YF, Gao Y, Chen B and Guan GX: Development and validation of a ferroptosis-related lncRNAs prognosis signature in colon cancer. Bosn J Basic Med Sci 21: 569-576, 2021.

15. Tang Y, Li C, Zhang YJ and Wu ZH: Ferroptosis-related long non-coding RNA signature predicts the prognosis of Head and neck squamous cell carcinoma. Int J Biol Sci 17: 702-711, 2021.

16. De Troyer L, Zhao P, Pastor T, Baietti MF, Barra J, Vendramin R, Dok R, Lechat B, Najm P, Van Haver D, et al: Stress-induced lncRNA LASTR fosters cancer cell fitness by regulating the activity of the U4/U6 recycling factor SART3. Nucleic Acids Res 48: 2502-2517, 2020.

17. Jiang $F$ and Shen $X$ : Current prevalence status of gastric cancer and recent studies on the roles of circular RNAs and methods used to investigate circular RNAs. Cell Mol Biol Lett 24: 53, 2019.

18. Dang Y, Ouyang X, Zhang F, Wang K, Lin Y, Sun B, Wang Y, Wang $L$ and Huang Q: Circular RNAs expression profiles in human gastric cancer. Sci Rep 7: 9060, 2017.

19. Fu D, Wang $\mathrm{C}, \mathrm{Yu} \mathrm{L}$ and $\mathrm{Yu} \mathrm{R}$ : Induction of ferroptosis by ATF3 elevation alleviates cisplatin resistance in gastric cancer by restraining Nrf2/Keap1/xCT signaling. Cell Mol Biol Lett 26: 26, 2021.

20. Gao Z, Deng G, Li Y, Huang H, Sun X, Shi H, Yao X, Gao L, Ju Y and Luo M: Actinidia chinensis Planch prevents proliferation and migration of gastric cancer associated with apoptosis, ferroptosis activation and mesenchymal phenotype suppression. Biomed Pharmacother 126: 110092, 2020.

21. Li C, Tian Y, Liang Y and Li Q: Circ_0008035 contributes to cell proliferation and inhibits apoptosis and ferroptosis in gastric cancer via miR-599/EIF4A1 axis. Cancer Cell Int 20: $84,2020$.

22. Zhao D, Zhang C, Jiang M, Wang Y, Liang Y, Wang L, Qin K, Rehman FU and Zhang X: Survival-associated alternative splicing signatures in non-small cell lung cancer. Aging (Albany NY) 12: 5878-5893, 2020.

23. Zhang H, Deng T, Liu R, Ning T, Yang H, Liu D, Zhang Q, Lin D, Ge S, Bai M, et al: CAF secreted miR-522 suppresses ferroptosis and promotes acquired chemo-resistance in gastric cancer. Mol Cancer 19: 43, 2020

24. $\mathrm{Lu} \mathrm{J}, \mathrm{Xu} F$ and $\mathrm{Lu} \mathrm{H}$ : LncRNA PVT1 regulates ferroptosis through miR-214-mediated TFR1 and p53. Life Sci 260: 118305, 2020.

25. Qi W, Li Z, Xia L, Dai J, Zhang Q, Wu C and Xu S: LncRNA GABPB1-AS1 and GABPB1 regulate oxidative stress during erastin-induced ferroptosis in HepG2 hepatocellular carcinoma cells. Sci Rep 9: 16185, 2019.

26. Wang M, Mao C, Ouyang L, Liu Y, Lai W, Liu N, Shi Y, Chen L, Xiao D, Yu F, et al: Long noncoding RNA LINC00336 inhibits ferroptosis in lung cancer by functioning as a competing endogenous RNA. Cell Death Differ 26: 2329-2343, 2019.

27. Yang Y, Tai W, Lu N, Li T, Liu Y, Wu W, Li Z, Pu L, Zhao X, Zhang $T$ and Dong Z: lncRNA ZFAS1 promotes lung fibroblast-to-myofibroblast transition and ferroptosis via functioning as a ceRNA through miR-150-5p/SLC38A1 axis. Aging (Albany NY) 12: 9085-1102, 2020.

28. Park MW, Cha HW, Kim J, Kim JH, Yang H, Yoon $\mathrm{S}$, Boonpraman N, Yi SS, Yoo ID and Moon JS: NOX4 promotes ferroptosis of astrocytes by oxidative stress-induced lipid peroxidation via the impairment of mitochondrial metabolism in Alzheimer's diseases. Redox Biol 41: 101947, 2021.

29. Yang RZ, Xu WN, Zheng HL, Zheng XF, Li B, Jiang LS and Jiang SD: Involvement of oxidative stress-induced annulus fibrosus cell and nucleus pulposus cell ferroptosis in intervertebral disc degeneration pathogenesis. J Cell Physiol 236: 2725-2739, 2021

30. Kou L, Sun R, Jiang X, Lin X, Huang H, Bao S, Zhang Y, Li C, Chen R and Yao Q: Tumor Microenvironment-responsive, multistaged liposome induces apoptosis and ferroptosis by amplifying oxidative stress for enhanced cancer therapy. ACS Appl Mater Interfaces 12: 30031-30043, 2020.

31. Ni S, Yuan Y, Qian Z, Zhong Z, Lv T, Kuang Y and Yu B: Hypoxia inhibits RANKL-induced ferritinophagy and protects osteoclasts from ferroptosis. Free Radic Biol Med 169: 271-282, 2021. 
32. Tang LJ, Zhou YJ, Xiong XM, Li NS, Zhang JJ, Luo XJ and Peng J: Ubiquitin-specific protease 7 promotes ferroptosis via activation of the p53/TfR1 pathway in the rat hearts after ischemia/reperfusion. Free Radic Biol Med 162: 339-352, 2021

33. Zhang Z, Guo M, Shen M, Kong D, Zhang F, Shao J, Tan S, Wang S, Chen A, Cao P and Zheng S: The BRD7-P53-SLC25A28 axis regulates ferroptosis in hepatic stellate cells. Redox Biol 36: 101619,2020

34. Shi Y, Zhu Y, Zheng X and Zheng Z: LINC00449 regulates the proliferation and invasion of acute monocytic leukemia and predicts favorable prognosis. J Cell Physiol 235: 6536-6547, 2020 .

35. Cai Q, Zhao X, Wang Y, Li S, Wang J, Xin Z and Li F: LINC01614 promotes osteosarcoma progression via miR-520a-3p/SNX3 axis. Cell Signal 83: 109985, 2021.

36. Chen Y, Cheng WY, Shi H, Huang S, Chen H, Liu D, Xu W, Yu J and Wang J: Classifying gastric cancer using FLORA reveals clinically relevant molecular subtypes and highlights LINC01614 as a biomarker for patient prognosis. Oncogene 40: 2898-2909, 2021.

37. Liu AN, Qu HJ, Yu CY and Sun P: Knockdown of LINC01614 inhibits lung adenocarcinoma cell progression by up-regulating miR-217 and down-regulating FOXP1. J Cell Mol Med 22: 4034-4044, 2018

38. Tang L, Chen Y, Peng X, Zhou Y, Jiang H, Wang G and Zhuang W: Identification and validation of potential pathogenic genes and prognostic markers in ESCC by integrated bioinformatics analysis. Front Genet 11: 521004, 2020.

39. Wang Y, Song B, Zhu L and Zhang X: Long non-coding RNA, LINC01614 as a potential biomarker for prognostic prediction in breast cancer. PeerJ 7: e7976, 2019.

40. Cheng J, Lou Y and Jiang K: Downregulation of long non-coding RNA LINC00460 inhibits the proliferation, migration and invasion, and promotes apoptosis of pancreatic cancer cells via modulation of the miR-320b/ARF1 axis. Bioengineered 12 96-107, 2021.

41. Hong H, Sui C, Qian T, Xu X, Zhu X, Fei Q, Yang J and $\mathrm{Xu}$ M: Long noncoding RNA LINC00460 conduces to tumor growth and metastasis of hepatocellular carcinoma through miR-342-3p-dependent AGR2 up-regulation. Aging (Albany NY) 12: 10544-10555, 2020.

42. Hou P, Meng S, Li M, Lin T, Chu S, Li Z, Zheng J, Gu Y and Bai J LINC00460/DHX9/IGF2BP2 complex promotes colorectal cancer proliferation and metastasis by mediating HMGA1 mRNA stability depending on m6A modification. J Exp Clin Cancer Res 40: 52, 2021.

43. Jiang Y, Cao W, Wu K, Qin X, Wang X, Li Y, Yu B, Zhang Z, Wang X, Yan M, et al: LncRNA LINC00460 promotes EMT in head and neck squamous cell carcinoma by facilitating peroxiredoxin-1 into the nucleus. J Exp Clin Cancer Res 38 $365,2019$.

44. Li F, Zhu W and Wang Z: Long noncoding RNA LINC00460 promotes the progression of cervical cancer via regulation of the miR-361-3p/Gli1 axis. Hum Cell 34: 229-327, 2021.

45. Cho SW, Xu J, Sun R, Mumbach MR, Carter AC, Chen YG, Yost KE, Kim J, He J, Nevins SA, et al: Promoter of lncRNA Gene PVT1 Is a tumor-suppressor DNA boundary element. Cell 173: 1398-1412.e22,2018.
46. Ghetti M, Vannini I, Storlazzi CT, Martinelli G and Simonetti G: Linear and circular PVT1 in hematological malignancies and immune response: Two faces of the same coin. Mol Cancer 19: 69, 2020.

47. Shigeyasu K, Toden S, Ozawa T, Matsuyama T, Nagasaka T, Ishikawa T, Sahoo D, Ghosh P, Uetake H, Fujiwara T and Goel A: The PVT1 lncRNA is a novel epigenetic enhancer of MYC, and a promising risk-stratification biomarker in colorectal cancer. Mol Cancer 19: 155, 2020.

48. Xu Y, Li Y, Jin J, Han G, Sun C, Pizzi MP, Huo L, Scott A, Wang Y, Ma L, et al: LncRNA PVT1 up-regulation is a poor prognosticator and serves as a therapeutic target in esophageal adenocarcinoma. Mol Cancer 18: 141, 2019.

49. Zhou C, Yi C, Yi Y, Qin W, Yan Y, Dong X, Zhang X, Huang Y, Zhang R, Wei J, et al: LncRNA PVT1 promotes gemcitabine resistance of pancreatic cancer via activating $\mathrm{Wnt} / \beta$-catenin and autophagy pathway through modulating the miR-619-5p/Pygo2 and miR-619-5p/ATG14 axes. Mol Cancer 19: 118, 2020.

50. Jiang Y, Li W, Yan Y, Yao X, Gu W and Zhang H: LINC01094 triggers radio-resistance in clear cell renal cell carcinoma via miR-577/CHEK2/FOXM1 axis. Cancer Cell Int 20: 274, 2020.

51. Jiang $\mathrm{Y}$, Zhang $\mathrm{H}$, Li W, Yan $\mathrm{Y}$, Yao $\mathrm{X}$ and $\mathrm{Gu} \mathrm{W}$ : FOXM1-activated LINC01094 promotes clear cell renal cell carcinoma development via MicroRNA 224-5p/CHSY1. Mol Cell Biol 40: e00357-19, 2020.

52. Li XX and Yu Q: Linc01094 accelerates the growth and metastatic-related traits of glioblastoma by sponging miR-126-5p. Onco Targets Ther 13: 9917-9928, 2020.

53. Xu H, Wang X, Wu J, Ji H, Chen Z, Guo H and Hou J: Long Non-coding RNA LINC01094 promotes the development of clear cell renal cell carcinoma by upregulating SLC2A3 via MicroRNA-184. Front Genet 11: 562967, 2020.

54. Xu J, Zhang P, Sun H and Liu Y: LINC01094/miR-577 axis regulates the progression of ovarian cancer. J Ovarian Res 13: $122,2020$.

55. Zhu B,Liu W, Liu H, Xu Q and Xu W: LINC01094 Down-regulates miR-330-3p and enhances the expression of MSI1 to promote the progression of glioma. Cancer Manag Res 12: 6511-6521, 2020.

56. Li Z, Li Y, Wang X, Liang Y, Luo D, Han D, Li C, Chen T, Zhang H, Liu Y, et al: LINC01977 promotes breast cancer progression and chemoresistance to doxorubicin by targeting miR-212-3p/GOLM1 Axis. Front Oncol 11: 657094, 2021.

57. Mei B, Wang Y, Ye W, Huang $H$, Zhou $Q$, Chen $Y$, Niu $Y$, Zhang $M$ and Huang Q: LncRNA ZBTB40-IT1 modulated by osteoporosis GWAS risk SNPs suppresses osteogenesis. Hum Genet 138: 151-166, 2019.

58. Zhao L, Ji G, Le X, Wang C, Xu L, Feng M, Zhang Y, Yang H, Xuan Y, Yang Y, et al: Long noncoding RNA LINC00092 acts in cancer-associated fibroblasts to drive glycolysis and progression of ovarian cancer. Cancer Res 77: 1369-1382, 2017.

This work is licensed under a Creative Commons Attribution-NonCommercial-NoDerivatives 4.0 International (CC BY-NC-ND 4.0) License. 